\title{
Medical Virtual Instrumentation for Personalized Health Monitoring: A Systematic Review
}

\author{
Olufemi Adeluyi and Jeong-A Lee \\ Department of Computer Engineering, Chosun University, Seosok Dong, \\ Gwangju, Korea
}

Submitted March 2015. Accepted for publication July 2015.

\begin{abstract}
The rising cost of healthcare and the increased senior population are some reasons for the growing adoption of the Personalized Health Monitoring (PHM) systems. Medical Virtual Instruments (MVIs) provide portable, flexible, and low-cost options for these systems. Our systematic literature search covered the Cochrane Library, Web of Science, and MEDLINE databases, resulting in 915 articles, and 25 of which were selected for inclusion after a detailed screening process that involved five stages. The review sought to understand the key aspects regarding the use of MVIs for PHM, and we identified the main disease domains, sensors, platforms, algorithms, and communication protocols for such systems. We also identified the key challenges affecting the level of integration of MVIs into the global healthcare framework. The review shows that MVIs provide a good opportunity for the development of low cost personalized health systems that meet the unique instrumentation requirements for a given medical domain.
\end{abstract}

Keywords: personalized health monitoring, medical instrumentation, telemetry, electrocardiogram (ECG), electroencephalography (EEG)

\section{INTRODUCTION}

Personalized Health Monitoring (PHM) refers to long term monitoring that is performed by a novice patient in an uncontrolled environment, such as his/her home [1]. It is a veritable tool that supports not only the monitoring of a patient's health status, but also the transition from a hospital-based, physician-centered healthcare delivery system, to one that is home-based and patient-centered. This transition has become necessary in the wake of challenges such as rising healthcare costs, dwindling healthcare budgets, a growing proportion of senior citizens in developed societies, and a growing need for medical systems personalized to suit the user specific needs $[2,3,4]$.

The feasibility and effectiveness of PHM depends on the availability of a pragmatic approach for providing medical instruments at the patient's home, similar to the traditional instruments found at a hospital. Unfortunately, many of these instruments are expensive

*Corresponding author: Jeong-A Lee, Department of Computer Engineering, Chosun University, 375 SeosukDong, Dong-Gu, Gwangju, 501-759, Korea. Phone: +82622307711. Fax: +82622307755. E-mail: jalee@chosun.ac.kr. Other author:adeluyi@chosun.kr. 
and inconveniently bulky, and providing them at the patient's home would nullify a number of potential benefits of PHM, including its low cost and portability. Virtual instruments can provide these tools to patients without forgoing the benefits of PHM.

A virtual instrument is an instrument that utilizes a hardware-software approach to system implementation. It takes advantage of the high performance of hardware and high flexibility of software to greatly reduce the size of the corresponding traditional instrument without sacrificing much of its functionality $[5,6]$. The hardware portion comprises the sensor, display, and memory, while the software part is made up of the processing and interface modules. These instruments can be deployed in several contexts and their specific use essentially depends on the creativity of the designer [7]. Medical Virtual Instruments (MVIs) are virtual instruments that are used within the context of medicine. They approach home-based health monitoring in a way that emphasizes system re-use, modularity, adaptability, and user-defined instrumentation. For this review, we are interested in MVIs that are used for PHM. These systems are expected to monitor patients' health and support medical tests in key domains of medicine [8-10].

The objective of this systematic review is to give an overview of the current body of work covering the use of virtual instruments for personalized health monitoring. The emphasis is on the identification of key architectures used for MVIs in terms of the type of sensors, architecture, modality, communication interface, and network model. Second, it describes the important application domains of MVI, its level of adaptation, and the common algorithms utilized. Third, it outlines the key outcomes of using MVIs for PHM as well as the current challenges and the anticipated future research direction for the field.

The remainder of this review is organized as follows:In Section 2, we present the Materials and Methods used to carry out the review. In Section 3, we outline the Results of the most common features of PHM-based medical instruments. We then discuss the results in Section 4, and conclude in Section 5.

\section{MATERIALS AND METHODS}

\subsection{Search Strategy}

A systematic literature search was performed to identify studies on MVI using the following databases: MEDLINE (1996-August 2014), Web of Science (1973-August 2014) and Cochrane Library (1992-August 2014). The relevant fields and research areas were identified and the following search query was used without limitations on the year or type of publication:

For MEDLINE:

Query: ("user-computer interface”[MeSH Terms] OR ("user-computer"] [All Fields] AND “interface"[All Fields]) OR "user-computer interface"[All Fields] OR ("virtual"[All Fields] AND "systems"[All Fields]) OR "virtual systems"[All Fields]) AND ("instrumentation"[Subheading] OR “instrumentation"[All Fields]) OR ("equipment and supplies" [MeSH Terms] OR ("equipment"[All Fields] AND "supplies"[All Fields]) OR "equipment and supplies"[All Fields] OR "device"[All Fields]) AND ("telemedicine”[MeSH Terms] OR "telemedicine”[All Fields]) AND Monitoring[All Fields]. 
For Web of Science and Cochrane Library:

Query: ("Virtual Systems" AND "Instrumentation" OR "Device” AND "Telemedicine" AND "Monitoring”).

The search resulted in 915 articles, with 642 from MEDLINE, 233 from Web of Science, and 40 from the Cochrane Library.

\subsubsection{Selection Process}

Our selection process was based on the following 5-stage strategy:

i. Deletion of doubles

ii. Title scan

iii. Abstract scan

iv. Cursory full text scan

v. Detailed full text scan

We started by deleting the 46 articles that were duplicated before a screening based on their titles. Titles that indicated contents different from research related to PHM and MVI were discarded. There were 573 articles discarded at this stage, leaving a total of 296 articles for the abstract-scan stage. During the abstract scan, we eliminated articles that did not align with the theme of our review, and 127 articles were filtered out at this stage.

The remaining 169 articles were subjected to a cursory scan of the full text, which essentially involved identifying the sections, as well as reading the introduction, discussion, and conclusion sections. Articles that did not give sufficient details on the items listed in our objectives were discarded. After this stage, there were 56 articles that were subjected to a detailed full text scan, and 39 of which were discarded. The criteria included those listed in the previous stage. A preference was also given to articles that covered multiple domains, those that addressed unique applications, and those that described the instrumentation process in some detail. After all these stages, we had 25 articles that were included in this review. A description of each of these articles is given in Appendix A. The search strategy is shown in Figure 1.

\section{RESULTS}

Based on the analysis of the 25 research articles included in this review, we identified a number of key features that characterize the use of MVIs for PHM. These features will be discussed in the subsequent subsections as follows:

- $\quad$ Architecture

- Application

- Outcomes

\subsection{Architecture of MVIs}

The architecture describes the hardware portion of the MVI and the communication interface. It comprises the sensors, system platform, and the communication interface utilized for both the local and remote ends of the MVI. 


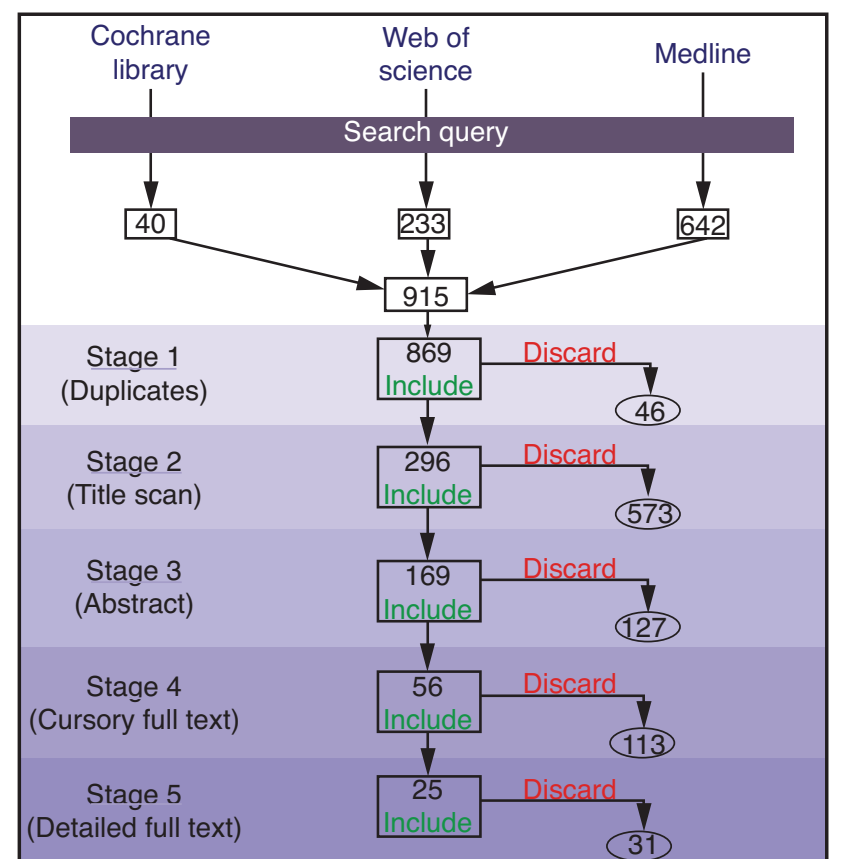

Figure 1. Search strategy.

\subsubsection{Sensors and Sensing}

The sensors capture the analog bio-signals from the patient and condition them for further processing. Table 1 shows the parameters and types of sensors used in the selected articles and the percentage of the 25 studies where they were used.

At $40.0 \%$, Electrocardiogram (ECG) sensors represent the most extensively used sensors for MVIs. As shown, when the Cardiac Implantable Electronic Device (CIED) sensors are included, the percentage rises to $48.0 \%$, implying that close to half of MVI systems monitor heart signals. Blood pressure (BP) sensors and accelerometers are next in prevalence at $24.0 \%$ each. It is interesting to note that a number of these sensors measure signals as a proxy for another signal of interest and are thus known as virtual sensors.

Virtual sensors [17,18] are quickly becoming an important part of the MVI architecture. They refer to sensors that are based on software rather than hardware and they infer their readings from the relevant hardware sensor(s). These virtual sensors enable patients to monitor bio-signals for which it is either impractical to have access to the signal of interest or for which the sensors or related equipment may be too expensive. From the reviewed articles, there were 12 cases, or $48.0 \%$, that used virtual sensing. The actual sensors used for this process are listed in Table 2. 
Table 1. Sensors and parameters measured in MVIs

\begin{tabular}{llcc}
\hline S/No. & Sensor & Cases & $\boldsymbol{\%}$ \\
\hline 1 & Electrocardiogram [11, 14, 20,21, 22, 23, 24, 27, 30,31] & 10 & 40.0 \\
2 & Blood pressure [11, 14, 21, 25, 27, 29] & 6 & 24.0 \\
3 & Accelerometer [14, 22, 26, 27, 29,32] & 6 & 24.0 \\
4 & Oxygen saturation [14, 21, 27, 29] & 4 & 16.0 \\
5 & Temperature [14, 25, 29,30] & 4 & 16.0 \\
6 & Microphone [15, 28, 30, 37] & 4 & 16.0 \\
7 & Posture [14,21, 29] & 3 & 12.0 \\
8 & Pressure [16,25,35] & 3 & 12.0 \\
9 & Weight [11,21] & 2 & 8.0 \\
10 & Cardiac implantable electronic device [12,19] & 2 & 8.0 \\
11 & Gyroscope [14,32] & 2 & 8.0 \\
12 & Blood glucose [33,36] & 2 & 8.0 \\
13 & Photodiode [29] & 1 & 4.0 \\
14 & Surface electromyography [13] & 1 & 4.0 \\
15 & Electroencephalography [34] & 1 & 4.0 \\
16 & Tilt [22] & 1 & 4.0 \\
17 & Camera [30] & 1 & 4.0 \\
18 & Pedometer [32] & 1 & 3.8 \\
19 & Gastrocnemius expansion [32] & 1 & 3.8 \\
21 & Electro dermal activity [37] & 1 & 3.8 \\
22 & Chest impedance [21] & 1 & 3.8 \\
\hline
\end{tabular}

Table 2. Examples of inferred parameters in MVIs

\begin{tabular}{|c|c|c|c|}
\hline S/No. & Inferred Parameters & Actual Sensor & No of Cases \\
\hline 1 & Respiratory rate $[14,2629,35]$ & ECG, accelerometer, pressure & 4 \\
\hline 2 & Heart rate $[29,31]$ & ECG & 2 \\
\hline 3 & Respiratory input impedance [16] & Pressure & 1 \\
\hline 4 & Drowsiness [37] & Electro dermal activity (EDA) & 1 \\
\hline \multirow[t]{2}{*}{5} & Gait analysis [32] & Gastrocnemius expansion & \\
\hline & & Measurement unit (GEMU) & 1 \\
\hline \multirow[t]{2}{*}{6} & Parkinson's disease & & \\
\hline & Progression $[15]$ & Microphone & 1 \\
\hline \multirow[t]{2}{*}{7} & Obstructive sleep apnea syndrome & & \\
\hline & $(\mathrm{OSAS})[28]$ & Microphone & 1 \\
\hline \multirow[t]{2}{*}{8} & Consciousness awareness [34] & EEG & 1 \\
\hline & Total & & 12 \\
\hline
\end{tabular}




\subsubsection{Platform}

Virtual instrumentation was introduced to the consumer market in the late 1980s by a company known as National Instruments through a product called LabVIEW (Laboratory Virtual Instrument Engineering Workbench). Other products currently available in the virtual instrumentation space include the Simulink software from Mathworks and the BioMobius [38] open source biomedical platform developed by the Technology Center for Independent Living (TRIL), Ireland. The Reconfigurable Virtual Instrumentation (RVI) open source platform of the International Center for Theoretical Physics (ICTP) in Trieste, Italy is another similar platform. It uses a Field Programmable Gate Array (FPGA) as its reconfiguration engine [39] and has been used to develop a neural monitoring system. Essentially, most of these virtual instruments are required to run on a dedicated general-purpose computer.

MVIs are developed to support PHM systems for which portability is an important requirement. Unlike the general virtual instrument approach, most MVIs do not use a $\mathrm{PC}$ as their platform, but instead a custom device [14-16, 20-23, 25-29, 32, 33-37] or a mobile phone/personal digital assistant (PDA), [11, 13, 24, 30, 31, 33] as shown in Table 3. The two cases of Cardiac Implantable Electronic Devices (CIEDs) [12,19] were not included since they only use mobile phones for communicating with a remote system.

\subsubsection{Network Models and Communication System}

All of the MVIs in the reviewed articles were based on a client-server network model. In most systems, the sensed signals were forwarded from the local (patient) end to an access point device in close proximity to the sensors for onward transmission to a remote server at the remote (physician) end. In many cases, the platforms described in the previous section are used as the access points.

One trend worth noting about MVI network models involves the direct connection between the output of the bio-signals and a remote webserver or cloud service, rather than a connection to a specific remote server on the physician's end. Five of the reviewed articles [22, 28, 31, 36, 37] used such a model. A number of advantages can be derived from this approach. One such advantage is the potential of "geographically decoupling" the bio-signals [36]. In other words, it reduces the mobility restrictions on

Table 3. Platforms and protocols used in MVIs

\begin{tabular}{|c|c|c|c|}
\hline \multicolumn{4}{|c|}{ Platforms } \\
\hline & Custom Devices & PDA/Phone & Laptop/PC \\
\hline Cases & 17 & 6 & 1 \\
\hline \multicolumn{4}{|c|}{ Local-MVI Communication Protocols } \\
\hline & Wireless & RS-232 & Others \\
\hline Cases & 17 & 2 & 3 \\
\hline \multicolumn{4}{|c|}{ Remote MVI Communication Protocols } \\
\hline & Cellular & WiFi & DSL \\
\hline Cases & 14 & 9 & 2 \\
\hline
\end{tabular}


the patients since their signals can be streamed to the webserver while they move about freely. Another advantage is that many different authorized personnel, such as the physician and the caregiver, can simultaneously view the patient's signals. The approach can also exploit the memory and processing capabilities of a web or cloud service while reducing the computational complexity of the MVI at the patient's end.

Communication via MVIs can either occur within the local patient modules or between the physician's remote MVI and the patient's local MVI. The communication in the former is known as local-MVI communication, while the latter is known as remote-MVI communication. The remote MVI is similar to the local MVI, with exception to the inclusion of the remote's bio-signal sensors. Much of its functionality involves the analysis of the signals, which results in a greater storage and processing capacity in the remote MVI.

The wireless protocol was the most common for local-MVI communication and was used in $73.9 \%$ of the cases (Table 3). This included $26.1 \%$ for Bluetooth $[11,21,30,31$, $33,34], 8.7 \%$ for ZigBee [22,24], and 39.1\% for unspecified wireless protocols [12, 19, $20,23,25,28,29,32,33]$. RS-232 protocols were used in $8.7 \%$ of the cases [14,37] and another $17.4 \%$ used other methods $[15,16,20,36]$. Some articles did not specify the local-MVI communication protocol [26, 27, 35].

The cellular networks were the most common approach for intra-MVI communication, used in 56\% of the cases [11-14, 19-21, 24, 27-30, 33, 36]. It was followed by the wireless approach at $36 \%, 28 \%$ of which were with Wi-Fi [13, 16, 20, $22,24,31,32]$ and the remaining $8 \%$ with unspecified wireless techniques $[16,23]$. DSL techniques were used in $8 \%$ of the references [16,21]. The details are shown in Table 3. Some articles used multiple techniques for remote-MVI communication while others failed to specify the local-MVI communication protocol $[17,25,26,27,34,35,37]$.

\subsection{Applications}

MVI applications refer to the disease domains, adopted modality, level of system adaptability, and algorithms that govern the operation of the instrument.

\subsubsection{Disease Domains}

MVIs can be used for, but are not limited to medical research applications, clinical applications, medical design development, healthcare information management systems, and mathematical modeling of physiologic systems [7]. However, from the analysis of the research articles in this review, we found that the use of MVIs for PHM focuses on certain specific disease domains. These domains are shown in Figure 2. The Cardiovascular Disease (CVD) domain accounts for over half of these cases. This is understandable since CVDs are the largest single contributor to global mortality [8]. The constituent monitoring scenarios classified under the cardiovascular domain are shown in Table 4.

Fitness monitoring refers to cases where the MVI did not target a specific domain. These cases were basically for monitoring general health and fitness. They accounted for about a quarter of the cases. The musculoskeletal domain addressed areas like fall 


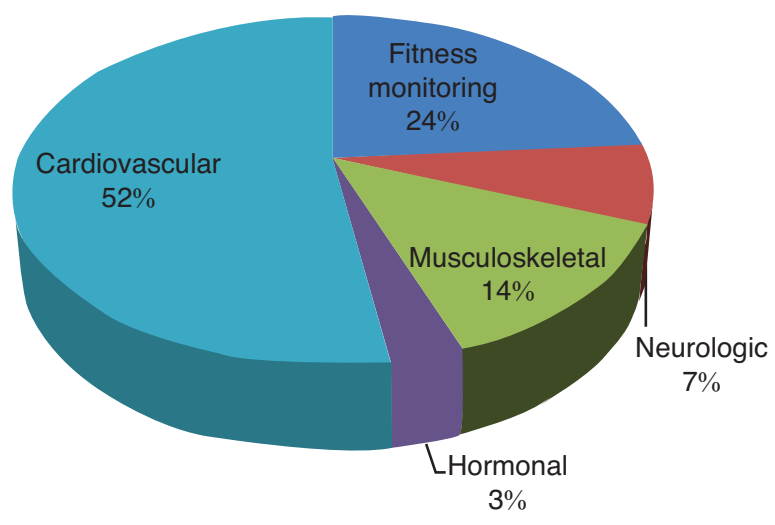

Figure 2. MVI disease domains.

Table 4. Monitoring scenarios for the cardiovascular domain

\begin{tabular}{llc}
\hline S/No. & Monitoring Scenario & No of Cases \\
\hline 1. & Heart monitoring [12, 19, 20, 23, 24, 31, 34, 35] & 8 \\
2. & Chronic heart failure [11,21] & 2 \\
3. & Spirometry [29] & 1 \\
4. & Hypertension [25] & 2 \\
5. & Blood flow velocity [18] & 1 \\
6. & Obstructive sleep apnea [28] & 1 \\
& Total & $\mathbf{1 5}$ \\
\hline
\end{tabular}

detection, gait analysis, and back pain. The cases classified under the neurologic domain focused on mental health monitoring using EEG sensors in the first case [24], and in the second case, an EDA sensor [27] for monitoring drowsiness. In a case involving the hormonal disease domain, blood glucose levels were monitored for the management of diabetes [23].

\subsubsection{Modality}

The modality refers to the expected effect on the state of health of the patient. $92.3 \%$ of the cases reviewed focused solely on extracting, analyzing, and reporting a patient's bio-signals. Only 2 MVI systems (7.7\%) triggered some form of therapeutic activity in response to the results of the analysis. The first case controlled the delivery of insulin to the diabetic patient [23] and the second case involved a stimulation to help keep the sleeping patient from snoring [28].

\subsubsection{System Adaptability}

Implicit in the name 'personalized health monitoring' is a need to have systems that are individually suited to the needs of a patient. This requires that the systems support some level of adaptability. The high level of flexibility in MVIs makes them a ready fit for such 
Table 5. Common algorithms used in MVIs

\begin{tabular}{lc}
\hline Algorithm & Cases \\
\hline Pan-Tompkins [21, 24, 27] & 3 \\
Peak detection [14, 26, 34] & 3 \\
Thresholding [14,15] & 2 \\
Wavelet transform [31,35] & 2 \\
Correlation [18] & 1 \\
Fuzzy-based [20] & 1 \\
Machine learning [30] & 1 \\
Least squares [16] & 1 \\
Edge detection [26] & 1 \\
\hline
\end{tabular}

a requirement. A total of $11(42.3 \%)$ of the reviewed articles supported some level of adaptation $[12,13,21,23,27,28,29,31,33,34,36]$, most of which were adaptation at the communication and architectural levels rather than at the patient level. Five of the six cases with adaptation at the subject level $[12,28,29,33,34]$ were based on the subject's clinical profile. The sixth case was based on the activity state of the subject [27].

\subsubsection{Algorithms}

Algorithms help patients and physicians make sense of the bio-signals generated by the subject. The type of disease domain and the amount of resources available on the MVI are some of the determinants of the type of algorithm used. Table 5 shows a list of the algorithms that were explicitly stated by the authors of the reviewed articles. The PanTompkins algorithm was featured in the most number of cases (3) and was used for MVIs targeting the cardiovascular domain, due to their real-time suitability and resource-light processing of ECG signals. Peak detection algorithms were also used in 3 of the MVIs.

\subsubsection{Outcomes/Results}

Some key outcomes were reported in the studies on the use of MVIs for PHM when compared to traditional medical instruments. These outcomes were based on the three areas listed below:

1. Effect on utility of the medical device. Common reported outcomes included:

a. Miniaturization of traditional medical instruments [13, 14, 16, 27, 28, 29, 32]

b. Reduced operator bias; enabling a more quantitative based analysis [13, 15, $33,34]$

c. Monitoring of device parameters [19]

2. Effect on healthcare. Common reported outcomes included:

a. Early detection of diseases $[11,12,21]$

b. Lower healthcare cost $[23,35,36]$

c. Reduced need for follow up and hospitalization $[11,12]$ 


\section{Effect on the perception and satisfaction of the patient:}

a. Patient feedback on usefulness of system [21,27]

Many of the studies reported that the MVI approach led to a positive effect on the utility of the medical devices $[11,12,13,14,15,16,19,21,23,27,28,29,32,33,34$, 35, 36]. Miniaturization was mentioned in these studies as the most important motivation for using MVIs. MVIs enable physicians to quantify the progression of disease and enable them to make informed choices that are not affected by the bias of operators that utilized equivalent traditional medical instruments. CIEDs and other implanted devices can be monitored with MVIs, as confirmed by one of the studies regarding the monitoring of leads, battery level, and device impedance [19].

The effect of MVIs on healthcare was another key category mentioned. Early detection of disease was the most important goal of the PHM systems mentioned in this category. Some studies also showed that the use of MVIs can lead to a reduction in the cost of the medical equipment and, by extension, also lead to a reduction in healthcare costs. Systems ranging anywhere between $\$ 1.000$ and $\$ 50,000$ could be replaced with a system costing approximately $\$ 25$. A $\$ 1,000,11$ sensor system could be replaced with a much cheaper, one sensor system [36]. Two studies also mentioned a reduction in the need for hospitalization and follow up appointments as an outcome for this category.

The third category of outcomes related to the perception and satisfaction of the patient. A few studies handed out questionnaires to the users of the system to assess the level of its perceived usefulness. In all of the reported cases, the patients found the systems useful.

\section{DISCUSSION}

This review discusses systems that have involved the use of medical virtual instruments for PHM. PHM systems can support the paradigm shift of global healthcare and its evolving focus from curative to preventive medicine. However, in order to ensure that PHM systems can support non-fitness related health monitoring, they must enable monitoring that addresses a wider range of health challenges. To achieve this, it is important to ensure that these systems incorporate sophisticated medical instruments. Unfortunately, such instruments tend to be complicated, large, and expensive, creating challenges for the PHM systems to provide simplicity, portability, and cost effectiveness. MVIs provide a veritable tool for bridging the gap between the needs of PHM systems and hospital-based health-monitoring systems that utilize traditional medical instruments.

Although the articles reviewed highlight several advantages of the MVI approach, much research is still needed in order to make MVI-based PHM systems attain a level of dependability and utility comparable to those offered by traditional medical instruments in the clinical setting. For one, on the issue of personalization, less than a quarter of the articles supported any kind of system adaptation that was based on the profile of the patient. Furthermore, the desired level of adaptability will be attained when MVIs can reconfigure themselves according to the genomic characteristics of the subject. In the same vein, MVIs need to advance beyond the current state of being used 
merely for patient monitoring. They need to start supporting therapeutic applications for PHM systems.

System reliability and quality of service need to be guaranteed for patients and physicians so that each can have the same level of confidence in MVIs as they do in traditional medical instruments. Issues like ensuring accurate medical alerts and, in effect, reducing false alarms are the initial areas that need to be addressed. MVIs are not as standardized as traditional medical instruments and may still require extended, and often complicated training sessions in order to provide reliable results. This can reduce the usability and adoption level of the systems. The approach in one of the studies involved the employment of an MVI that reduced the need for system training to the barest minimum [34]. Such an approach can aid in the evolution and acceptance of MVIs.

The high levels of sensitivity of health records make privacy, security, and authentication issues of prime importance. Six of the reviewed articles $(23.1 \%$ of the cases) used encryption techniques to protect patient data. Another article [33] investigated an interesting concept of e-consents to support privacy. Virtual sensing can provide an interesting approach to privacy and confidentiality. For instance, a cloud service library containing several MVIs can be developed with the ability to screen for different types of diseases. An interested subject can then use a virtual sensing approach to generate a proxy signal without having to visit the hospital to get the standard biosignals required for the given test. The subject can then upload this signal to the disease search engine and retrieve the results that indicate the presence or absence of the diseases that were screened for. In order to increase confidentiality, the system can be developed to use the biometrics of the subject for the bio-signal upload and the result download processes.

The requirement for mobility in PHM systems comes with a need for flexible and less power- hungry applications. Generally, these systems use batteries as their power sources. However, continuous real-time monitoring easily drains batteries. Alternative power options can enhance the utility value of MVIs. For example, power-harvesting techniques prove to be useful. Other options include power via wireless magnetic telemetry. [25].

More research is needed to expand the variety of MVIs beyond the systems that mainly focus on cardiovascular health. For example, mental health is becoming a global healthcare concern. It has been reported that one in four people now experience a mental health issue in their lifetime [9]. Surprisingly, only one of the reviewed studies that used MVIs were based on bio-signals from the brain [34]. Researchers should be taking advantage of the low-cost option provided by EEG sensors to monitor brain signals. There are encouraging signs that MVIs are growing in scope and sophistication [40]. Examples include their use for biomedical imaging [41], monitoring Parkinson's disease $[42,43]$, and respiratory disease $[44,45]$.

Non-invasiveness and non-intrusiveness are two words that describe the desired type of sensors for MVIs. The sensors play an important role in determining the quality of the signals being monitored. However, bio-signals from a number of the non-invasive or non-intrusive sensors do not generally provide health information as detailed as those from invasive tests performed at a hospital. For example, 644 tests are performed with 
body fluids [10]. One would not expect MVIs to carry out as many tests, but it still shows that greatly increasing the number of possible tests would aid in the development of MVIs. Virtual sensing provides an opportunity to use the current non-invasive biosignals to simulate the more invasive bio-signals. For example, one study obviates the need for expensive and complex polysomnography equipment by inferring its readings from a virtual sensor [35]. The virtual sensor was based on a pressure sensor embedded in a pillow. Also, capacitive sensing techniques mentioned in [31] can be useful in the development of more non-intrusive sensors.

A typical healthcare workflow includes, but is not limited to aspects like diagnosis, decision-making, treatment, and administrative procedures [46]. The use of MVIs would affect at least two of these aspects-most likely diagnosis and decision-making. As such, it may be necessary to redesign the healthcare workflow and organizational models to accommodate the use of MVIs. In a similar vein, reimbursement schemes need to include workflows that are based on the use of MVIs.

The results of this work are in line with some of the orientations described in European Community's report entitled "COMMUNICATION FROM THE COMMISSION TO THE EUROPEAN PARLIAMENT, THE COUNCIL, THE EUROPEAN ECONOMIC AND SOCIAL COMMITTEE AND THE COMMITTEE OF THE REGIONS-on telemedicine for the benefit of patients, healthcare systems and society" [47]. For instance, the report mentioned the importance of integrating telemedicine into the healthcare workflow. It also identifies the support of seniors, the reduction of hospital visits, and the reduction in healthcare costs as potential benefits of telemedicine and health monitoring.

One more thing to note about the challenges and future perspectives of MVI in PHM relates to the potential of webservers as a tool for decoupling the monitoring process from the limitations imposed by location. By taking advantage of miniaturized integrated circuits and microprocessors, MVIs can provide sensors that immediately transfer the signals to an always-on, local or remote webserver. This would enable realtime monitoring and allow all authorized persons to simultaneously view the signals. It would also allow MVIs to take advantage of the huge memory and processing capabilities of cloud computing.

There are a number of limitations in this review. First, the studies were based on projects managed by universities and other research institutes, not by hospitals. As such, not many of the covered systems have become mainstream commercial solutions continuously deployed in real-life environments. Second, there were few clinical trials and many were simulation studies. For those assessed with human subjects, many had small sample sizes and were based on a short test period. However, a number of trials involving CIEDs had large sample sizes. For example, 1650 patients in 75 Italian centers were monitored for periods ranging from 10-31 months [48].

Thirdly, only three of the studies gave details of the cost of implementation, making it difficult to directly compare with equivalent traditional medical instruments. Furthermore, with the rapid evolution of medical research and technology, there could be many implemented projects whose findings have not yet been published. As such, it is likely that this review has underestimated some of the current applications and techniques involving MVIs. 


\section{CONCLUSIONS}

In this systematic review, we have identified the main modules of PHM-oriented instrumentation and the issues that determine their utility. To a large extent, current PHM systems are mainly used for fitness monitoring and niche applications. This should change, and PHM instrumentation should support a greater level of adaptability and dependability in order to become a system of choice in today's healthcare tool chain. The review shows that research on the use of MVIs in PHM is still in its infancy with many challenges to overcome. However, despite the challenges, it holds the unique promise of providing the patients with customizable medical instrumentation, at an affordable cost, in the comfort of their homes.

\section{ACKNOWLEDGEMENT}

This work was supported in part by research funds from Chosun University (2014-2015).

\section{CONFLICT OF INTEREST}

The authors indicate no potential conflicts of interest in this work.

\section{REFERENCES}

[1] Pärkkä J. Analysis of Personal Health Monitoring Data for Physical Activity Recognition and Assessment of Energy Expenditure, Mental Load and Stress. PhD Dissertation, Tampere University of Technology, 2011.

[2] Jayadevappa R and Chhatre S. Patient Centered Care - A Conceptual Model and Review of the State of the Art. The Open Health Services and Policy Journal, 2011, 4:5-25.

[3] Billis A, Papageorgiou E, Frantzidis C, Tsatali M, Tsolaki A, Bamidis P. A Decision-Support Framework for promoting Independent Living and Ageing Well. IEEE Journal of Biomedical and Health Informatics. 2014 Jul 25.

[4] Díaz-Rodríguez N, Cadahía OL, Cuéllar MP, Lilius J and Calvo-Flores MD. Handling real-world context awareness, uncertainty and vagueness in real-time human activity tracking and recognition with a fuzzy ontology-based hybrid method. Sensors (Basel). 2014, 14(10):18131-71. doi: 10.3390/s141018131.

[5] Qiua X-J, Zhengb W-H, Tanga Y-T and Lua F. The Test Verification Design Method Based on Rapid Prototyping Technology of Aero-engine. Procedia Engineering. 2015, 99:981-990.

[6] Adeluyi $\mathrm{O}$ and Lee J-A. Medical Virtual Instrumentation for Ambient Assisted Living: Part 1 Concepts. Measurement and Control Journal. 2015, 48(6):167-177.

[7] Olansen JB and Rosow E. Virtual Bio-Instrumentation: Biomedical, Clinical, and Healthcare Applications in LabVIEW. Prentice Hall PTR, New Jersey 2001.

[8] Promoting Cardiovascular Health in the Developing World: A Critical Challenge to Achieve Global Health. Institute of Medicine (US) Committee on Preventing the Global Epidemic of Cardiovascular Disease: Meeting the Challenges in Developing Countries, Fuster V, Kelly BB (Eds). Washington (DC): National Academies Press (US), 2010.

[9] Votruba N and Thornicroft G. The importance of mental health in the Sustainable Development Goals. BJPsych International 2015,12(1):2-4.

[10] A-Z list of laboratory tests at the Central Manchester University Hospitals, United Kingdom, www.cmft.nhs.uk/info-for-health-professionals/laboratory-medicinela-z-list-of-laboratory-tests. Accessed June 3, 2015. 
[11] Koehler F, Winkler S, Schieber M, Sechtem U, Stangl K, Böhm M, Boll H, Baumann G, Honold M, Koehler K, Gelbrich G, Kirwan BA and Anker SD. Impact of remote telemedical management on mortality and hospitalizations in ambulatory patients with chronic heart failure: the telemedical interventional monitoring in heart failure study. Circulation. 2011, 123(17):1873-80. doi: 10.1161/CIRCULATIONAHA.111.018473.

[12] Ricci RP, Morichelli L and Santini M. Remote control of implanted devices through Home Monitoring technology improves detection and clinical management of atrial fibrillation. Europace 2009, 11(1):54-61. doi:10.1093/europace/eun303.

[13] Guerri JC, Antón AB, Pajares A, Monfort M and Sánchez D. A mobile device application applied to low back disorders. Multimedia Tools and Applications. 2009, 42(3):317-340.

[14] Kang JM, Yoo T and Kim H-C. A Wrist-Worn Integrated Health Monitoring Instrument with a TeleReporting Device for Telemedicine and Telecare. IEEE Transactions on Instrumentation and Measurement.2006,55(5):1655-1662.

[15] Tsanas A, Little MA, McSharry PE and Ramig LO. Accurate telemonitoring of Parkinson's disease progression by noninvasive speech tests. IEEE Transactions on Biomedical Engineering. 2010, 57(4):884-93. doi:10.1109/TBME.2009.2036000.

[16] Dellacà RL, Gobbi A, Pastena M, Pedotti A and Celli B. Home monitoring of within-breath respiratory mechanics by a simple and automatic forced oscillation technique device. Physiological Measurements. 2010, 31(4):N11-24. doi:10.1088/0967-3334/31/4/N01.

[17] Harini M, Bhairavi K, Gopicharan R, Ganapathy K and Vaidehi V. Virtualization of healthcare sensors in cloud. 2013 International Conference on Recent Trends in Information Technology (ICRTIT). 2013: 663-667.

[18] Madria S, Kumar V and Dalvi R. Sensor Cloud: A Cloud of Virtual Sensors. IEEE Software. 2014, 31(2):70-77, DOI Bookmark: http://doi.ieeecomputersociety.org/10.1109/MS.2013.141.

[19] Sticherling C, Kühne M, Schaer B, Altmann D and Osswald S. Remote monitoring of cardiovascular implantable electronic devices: prerequisite or luxury? Swiss Medical Weekly. 2009, 139(41-42):596-601. doi:smw-12667

[20] Wang H, Peng D, Wang W, Sharif H, Chen H-H and Khoynezhad AA. Resource-aware secure ECG healthcare monitoring through body sensor networks. IEEE Wireless Communications. 2010, 17(1): 12-19.

[21] Fanucci L, Saponara S, Bacchillone T, Donati M, Barba P, Sánchez-Tato I and Carmona C. Sensing Devices and Sensor Signal Processing for Remote Monitoring of Vital Signs in CHF Patients. IEEE Transactions on Instrumentation and Measurement. 2013, 62(3):553-569.

[22] Lee S-J, Kim J and Lee M. The Design of the m-Health Service Application Using a Nintendo DS Game Console. Telemedicine and e-Health 2011, 17(2):124-30. doi:10.1089/tmj.2010.0080. Epub 2011 Jan 9.

[23] Dilmaghani RS, Bobarshad H, Ghavami M, Choobkar S and Wolfe C. Wireless sensor networks for monitoring physiological signals of multiple patients. IEEE Transactions on Biomedical Circuits and Systems. 2011, 5(4):347-56. doi:10.1109/TBCAS.2011.2114661.

[24] Hii P-C and Chung W-Y. A Comprehensive Ubiquitous Healthcare Solution on an Android ${ }^{\mathrm{TM}}$ Mobile Device. Sensors (Basel). 2011,11(7):6799-6815. Published online Jun 29, 2011. doi: 10.3390/s110706799, PMCID: PMC3231662.

[25] Cleven NJ, Müntjes JA, Fassbender H, Urban U, Görtz M, Vogt H, Gräfe M, Göttsche T, Penzkofer T, Schmitz-Rode T and Mokwa W. A novel fully implantable wireless sensor system for monitoring hypertension patients. IEEE Transactions on Biomedical Engineering. 2012, 59(11):3124-30. doi: 10.1109/TBME.2012.2216262.

[26] Pitts DG, Patel MK, Lang PO, Sinclair AJ and Aspinall R. A respiratory monitoring device based on clavicular motion. Physiological Measurements. 2013, 34(8):N51-61. doi: 10.1088/0967-3334/34/8/N51. 
[27] Anliker U, Ward JA, Lukowicz P, Tröster G, Dolveck F, Baer M, Keita F, Schenker EB, Catarsi F, Coluccini L, Belardinelli A, Shklarski D, Alon M, Hirt E, Schmid R and Vuskovic M. AMON: a wearable multiparameter medical monitoring and alert system. IEEE Transactions on Information Technology in Biomedicine. 2004, 8(4):415-27.

[28] Cheng CM, Hsu YL, Young CM and Wu CH. Development of a portable device for telemonitoring of snoring and obstructive sleep apnea syndrome symptoms. Telemedicine and e-Health. 2008, 14(1):55-68. doi: 10.1089/tmj.2007.0022.

[29] Chun H, Kang J, Kim KJ, Park KS and Kim HC. IT-based diagnostic instrumentation systems for personalized healthcare services. Studies in Health Technology and Informatics. 2005, 117:180-90.

[30] Yu Y, Li J and Liu J. M-HELP: a miniaturized total health examination system launched on a mobile phone platform. Telemedicine and e-Health. 2013, 19(11):857-65. doi:10.1089/tmj.2013.0031.

[31] Fong E-M and Chung W-Y. Mobile Cloud-Computing-Based Healthcare Service by Noncontact ECG Monitoring. Sensors (Basel). 2013, 13(12):16451-16473. doi:10.3390/s131216451.

[32] Giansanti D, Morelli S, Maccioni G and Grigioni M. Portable kit for the assessment of gait parameters in daily telerehabilitation. Telemedicine and e-Health. 2013,19(3):224-32. doi: 10.1089/tmj.2012.0091.

[33] Gómez EJ, Hernando Pérez ME, Vering T, Rigla Cros M, Bott O, García-Sáez G, Pretschner P, Brugués E, Schnell O, Patte C, Bergmann J, Dudde R and de Leiva. A. The INCA system: a further step towards a telemedical artificial pancreas. IEEE Transactions on Information Technology in Biomedicine. 2008, 12(4):470-9. doi:10.1109/TITB.2007.902162.

[34] D'Arcy RC, Hajra SG, Liu C, Sculthorpe LD and Weaver DF. Towards brain first-aid: a diagnostic device for conscious awareness. IEEE Transactions on Biomedical Engineering. 2011, 58(3):750-4. doi: 10.1109/TBME.2010.2090880. Epub 2010 Nov 11.

[35] Chen W, Zhu X, Nemoto T, Kitamura K, Sugitani K and Wei D. Unconstrained monitoring of longterm heart and breath rates during sleep. Physiological Measurements. 2008, 29(2):N1-10. doi: 10.1088/0967-3334/29/2/N01.

[36] Nemiroski A, Christodouleas DC, Hennek JW, Kumar AA, Maxwell EJ, Fernández-Abedul MT and Whitesides GM. Universal mobile electrochemical detector designed for use in resource-limited applications. Proceedings of the National Academy of Sciences of the United States of America. 2014, 111(33):11984-11989, Bell AT (ed). doi: 10.1073/pnas.1405679111.

[37] Lee Y, Lee B and Lee M. Wearable sensor glove based on conducting fabric using electrodermal activity and pulse-wave sensors for e-health application. Telemedicine and e-Health, 2010, 16(2):209-17. doi:10.1089/tmj.2009.0039.

[38] The BioMobius Platform. http://www.capsil.org/capsilwiki/index.php/BioMOBIUS. Accessed June 2 2015.

[39] The Reconfigurable Virtual Instrument FPGA Platform. http://mlab.ictp.it/rvi/system.html. Accessed June 22015.

[40] Special Issue on Mobile Medicine. Annals of Biomedical Engineering. 2014, 42(11):2203-2204.

[41] Roy M, Seo D, Oh CH, Nam MH, Kim YJ and Seo S. Low-cost telemedicine device performing cell and particle size measurement based on lens-free shadow imaging technology. Biosensors and Bioelectronics. 2015, 15,67:715-23. doi:10.1016/j.bios.2014.10.040.

[42] Barroso MC, Esteves GP, Nunes TP, Silva LMG, Faria ACD and Melo PL. A telemedicine instrument for remote evaluation of tremor: design and initial applications in fatigue and patients with Parkinson's Disease BioMedical Engineering OnLine. 2011, 10:14. doi:10.1186/1475-925X-10-14.

[43] Patel S, Chen BR, Buckley T, Rednic R, McClure D, Tarsy D, Shih L, Dy J, Welsh M, Bonato P. Home monitoring of patients with Parkinson's disease via wearable technology and a web-based application. 2010 Conf Proc IEEE Eng Med Biol Soc. 2010, 4411-4. doi:10.1109/IEMBS.2010.5627124. 
[44] da Silva Junior EP, Esteves GP, Dames KK, Melo PL. A telemedicine instrument for Internet-based home monitoring of thoracoabdominal motion in patients with respiratory diseases. Review of Scientific Instruments. 2011, 82(1):014301. doi:10.1063/1.3529443.

[45] Silva Junior EP1, Esteves GP, Faria AC, Melo PL. An internet-based system for home monitoring of respiratory muscle disorders. IEEE Eng Med Biol Soc Conf Proc. 2010,2010:5492-5. doi: 10.1109/IEMBS.2010.5626581.

[46] Macedo M and Isais P. Standards Related to Interoperability in EHR \& HS, in: Sicilia M.A. and Balazote P (eds). Interoperability in Healthcare Information Systems: Standards, Management, and Technology: Standards, Management, and Technology. 2013:19-44.

[47] COMMUNICATION FROM THE COMMISSION TO THE EUROPEAN PARLIAMENT, THE COUNCIL, THE EUROPEAN ECONOMIC AND SOCIAL COMMITTEE AND THE COMMITTEE OF THE REGIONS - on telemedicine for the benefit of patients, healthcare systems and society. http://www.ipex.eu/IPEXL-WEB/dossier/dossier.do? code $=C O M \&$ year $=2008 \&$ number $=0689$. Accessed June 12015.

[48] Ricci RP, Morichelli L, D’Onofrio A, Cal\&ograve; L, Vaccari D, Zanotto G, Curnis A, Buja G, Rovai N, Gargaro A. Manpower and Outpatient Clinic Workload for Remote Monitoring of Patients with Cardiac Implantable Electronic Devices: Data from the HomeGuide Registry. Journal of Cardiovascular Electrophysiology. 2014, 25(11):1216-23. 


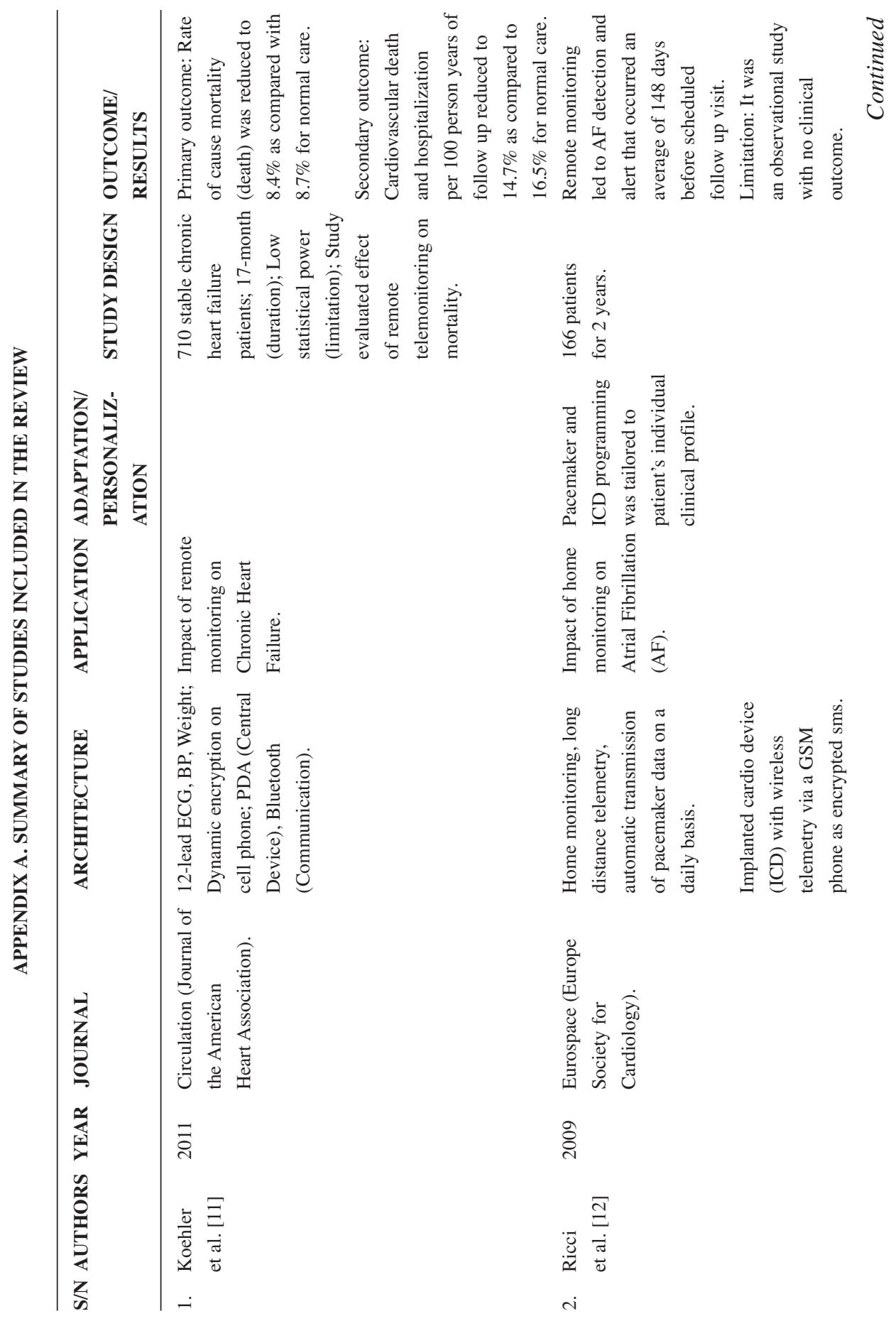




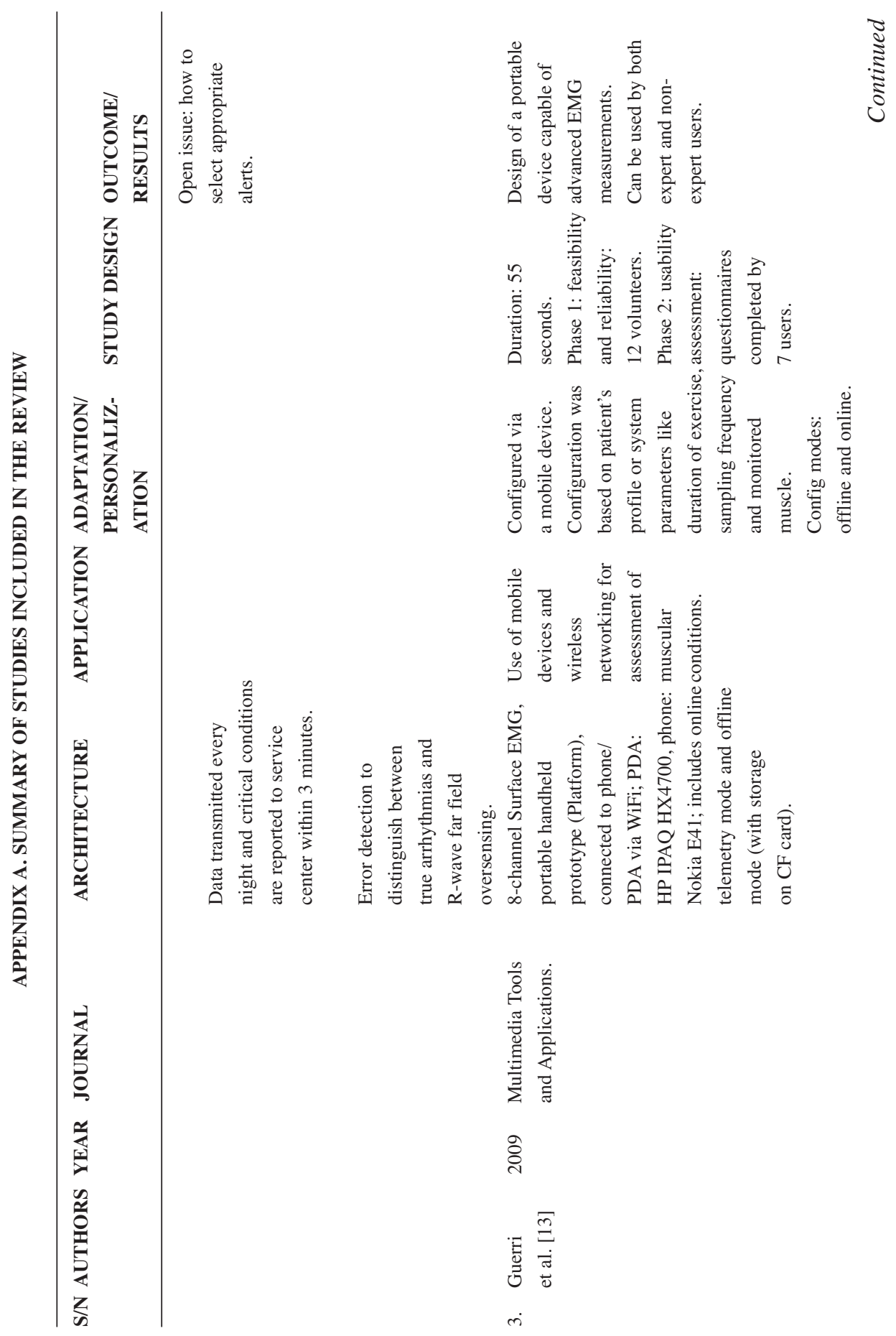




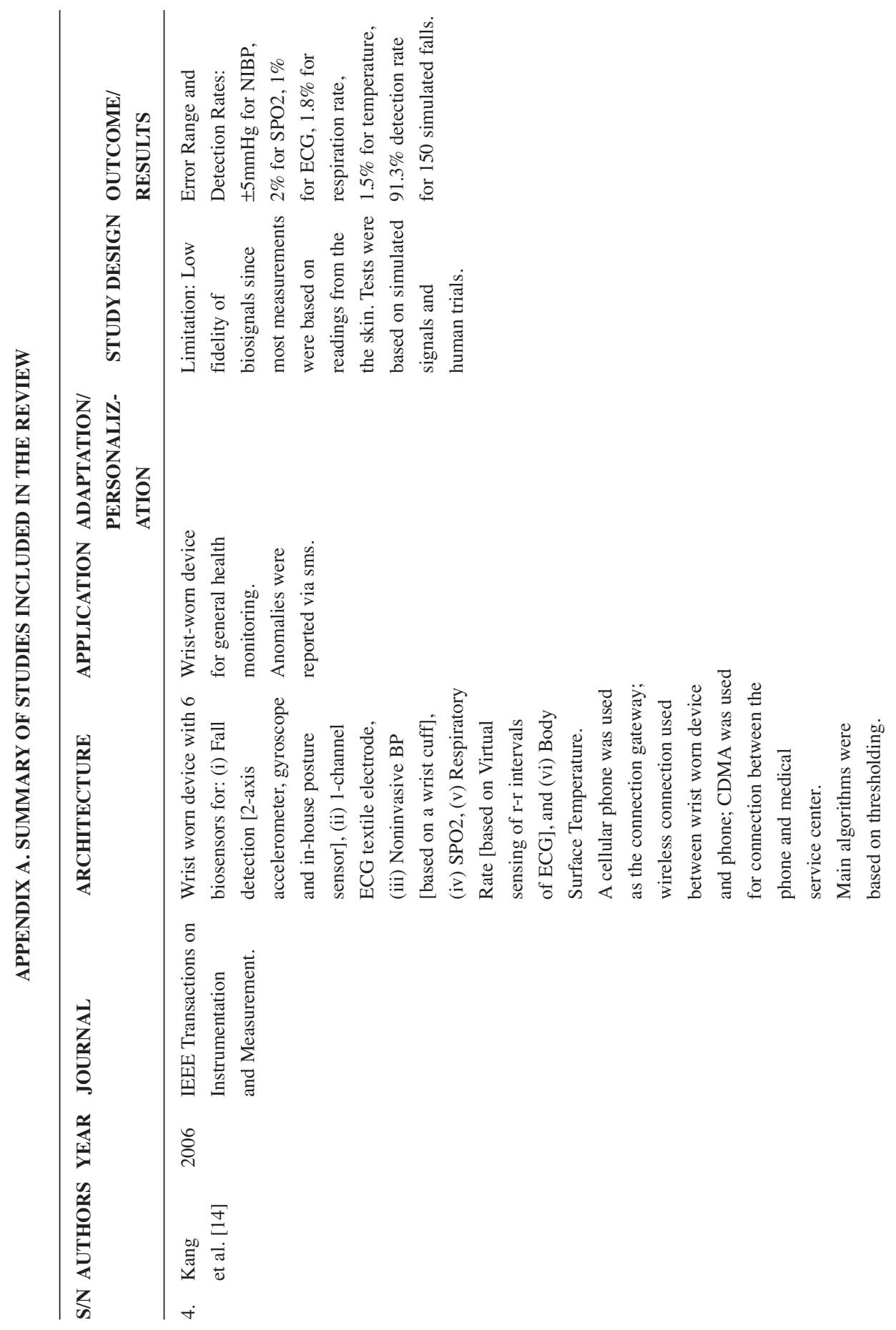




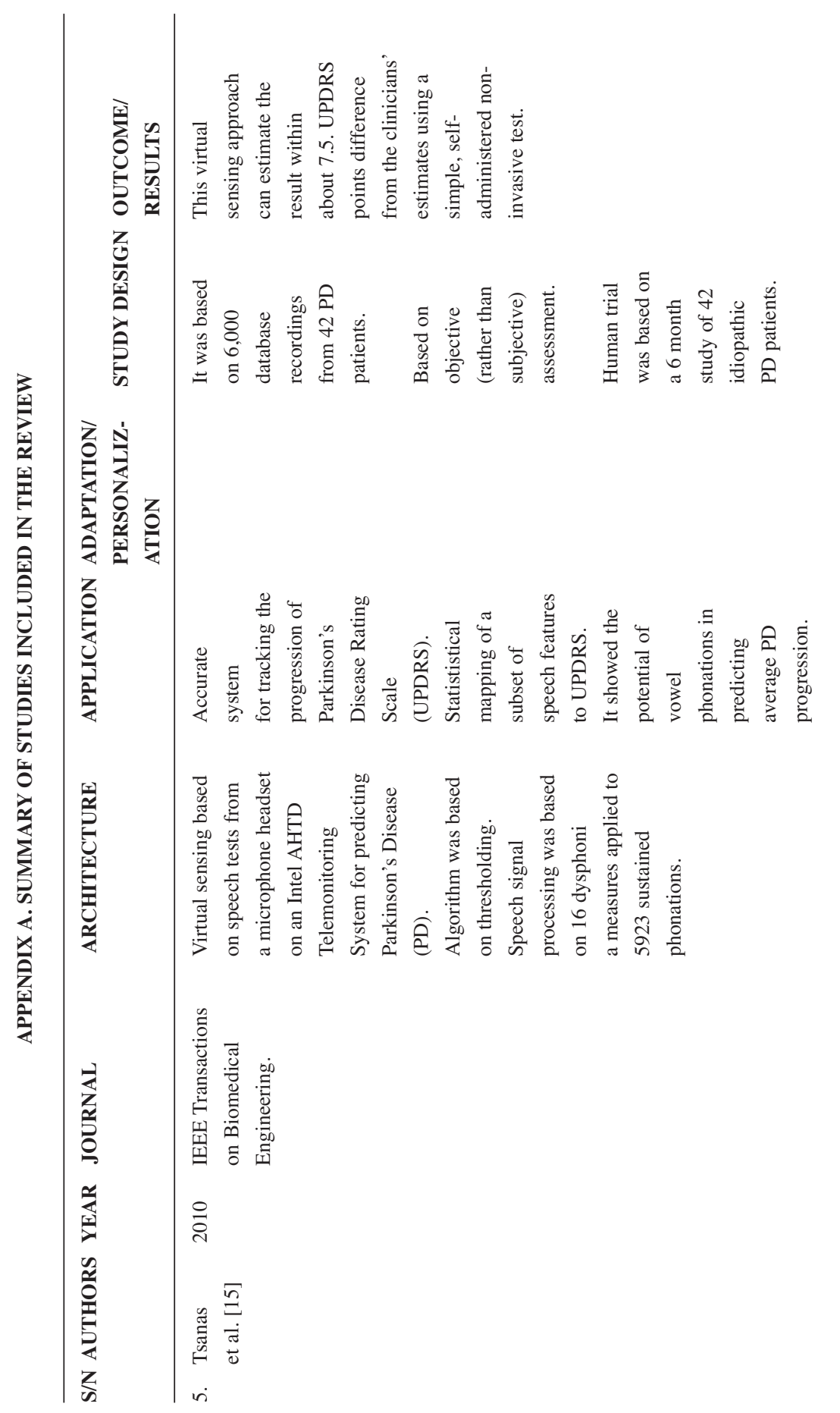

טัฐ 


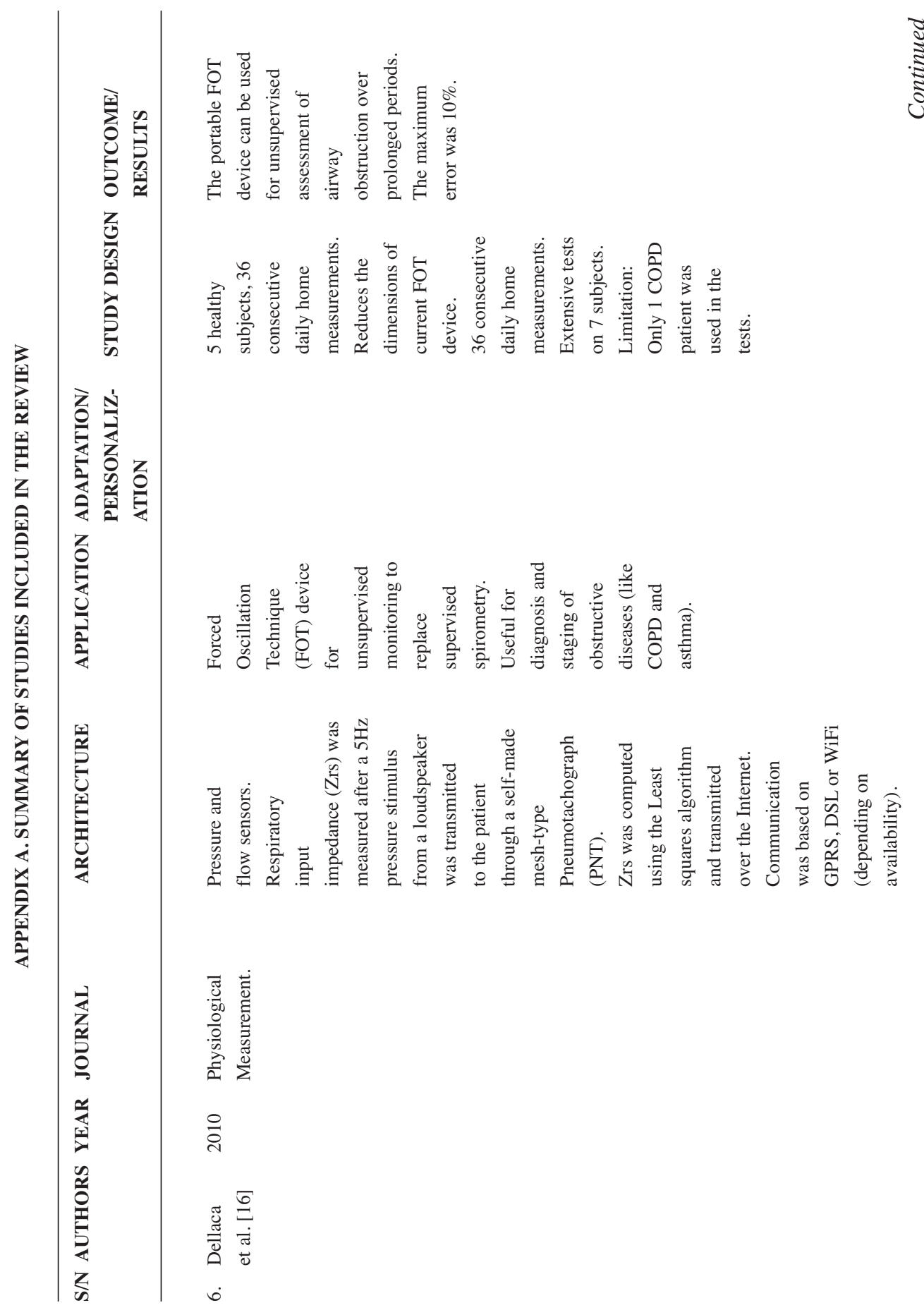




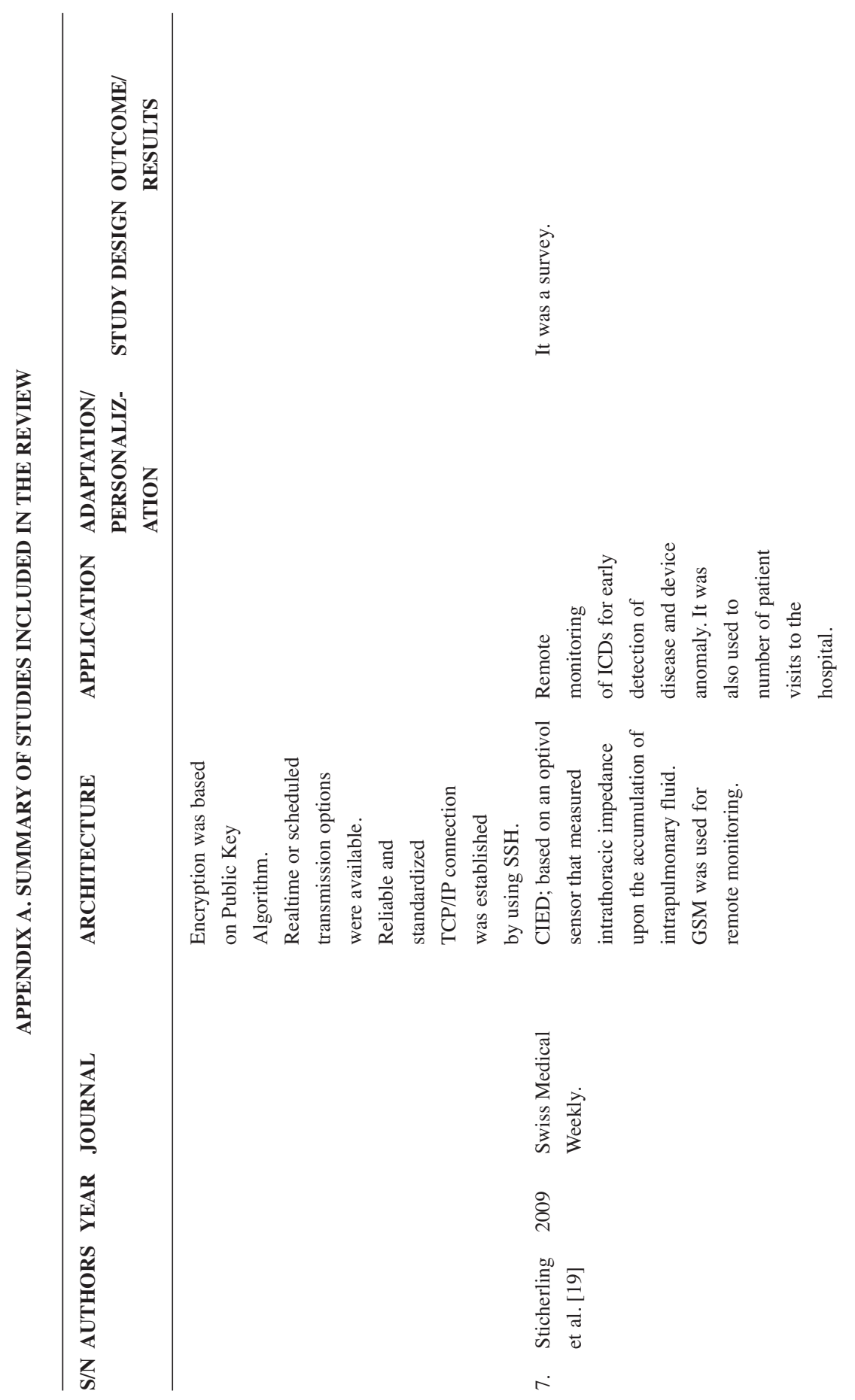




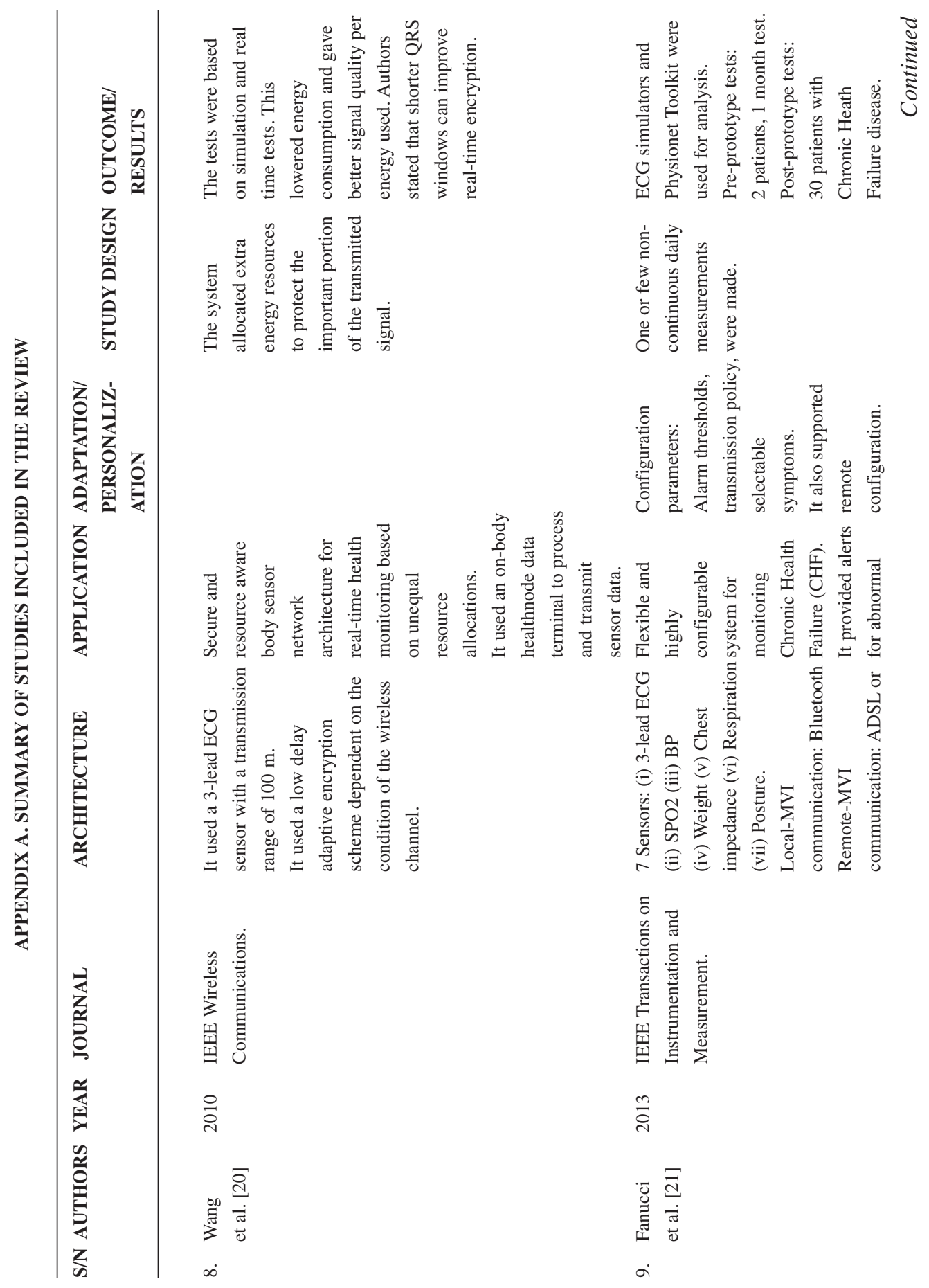




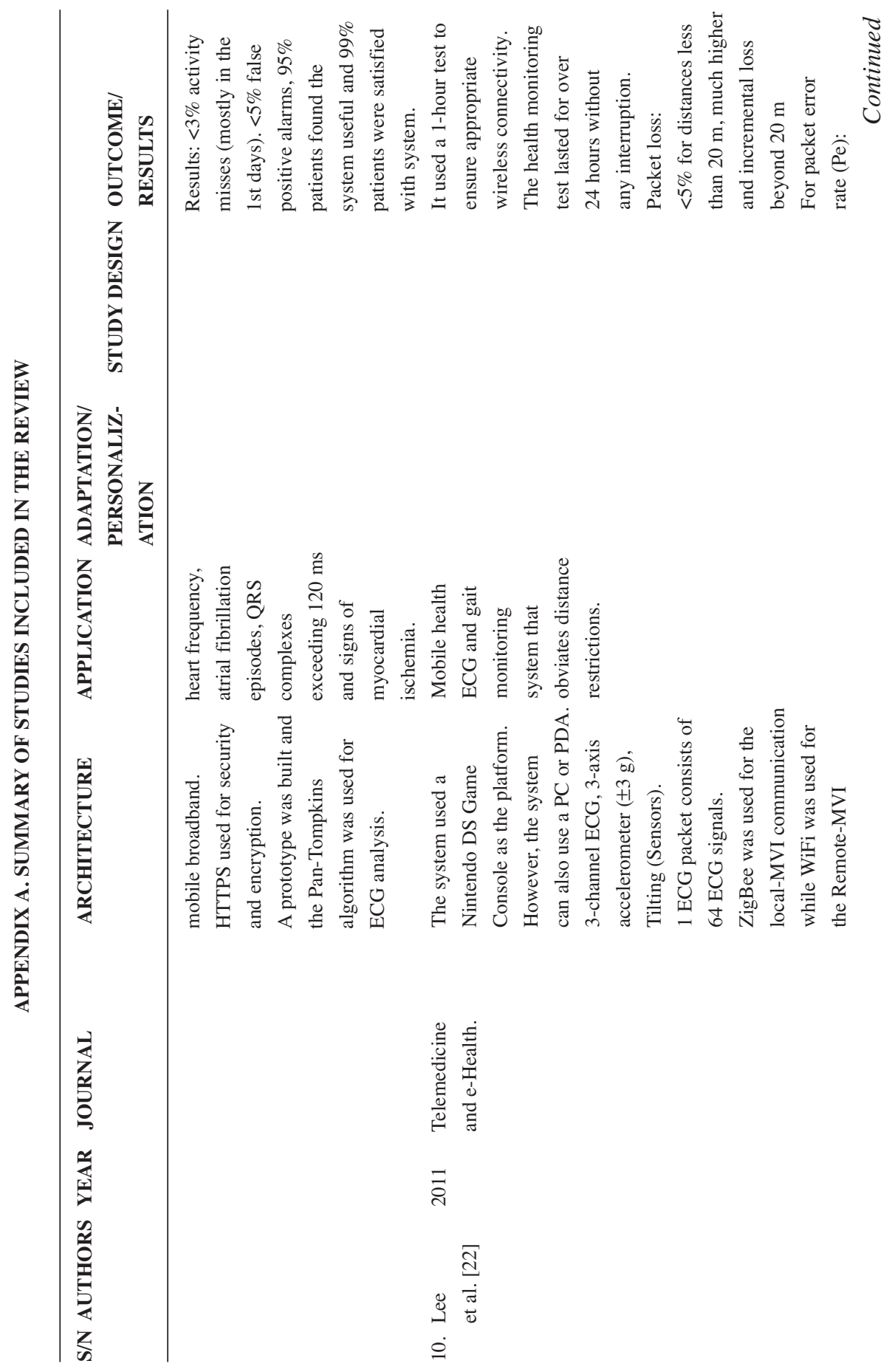




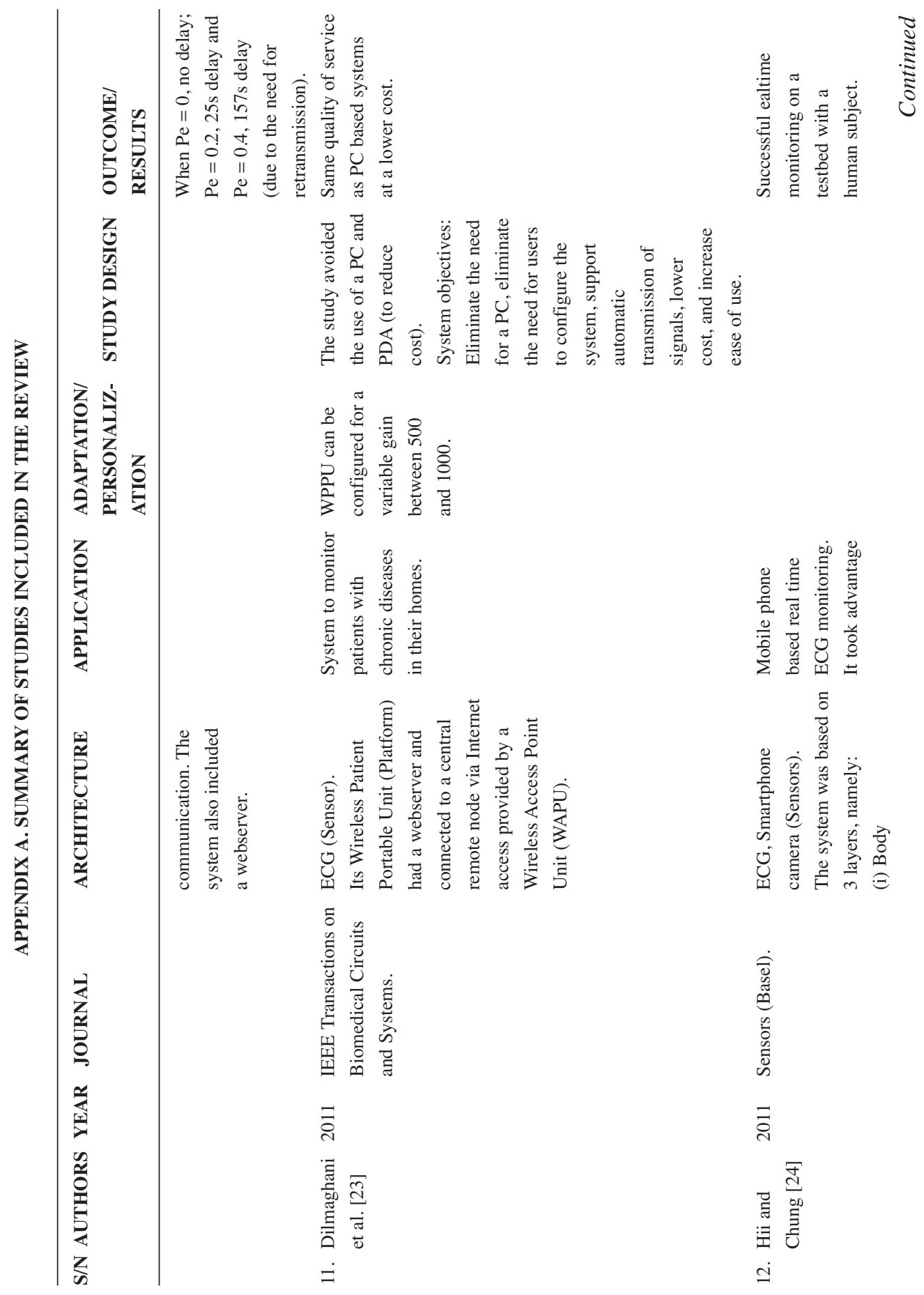




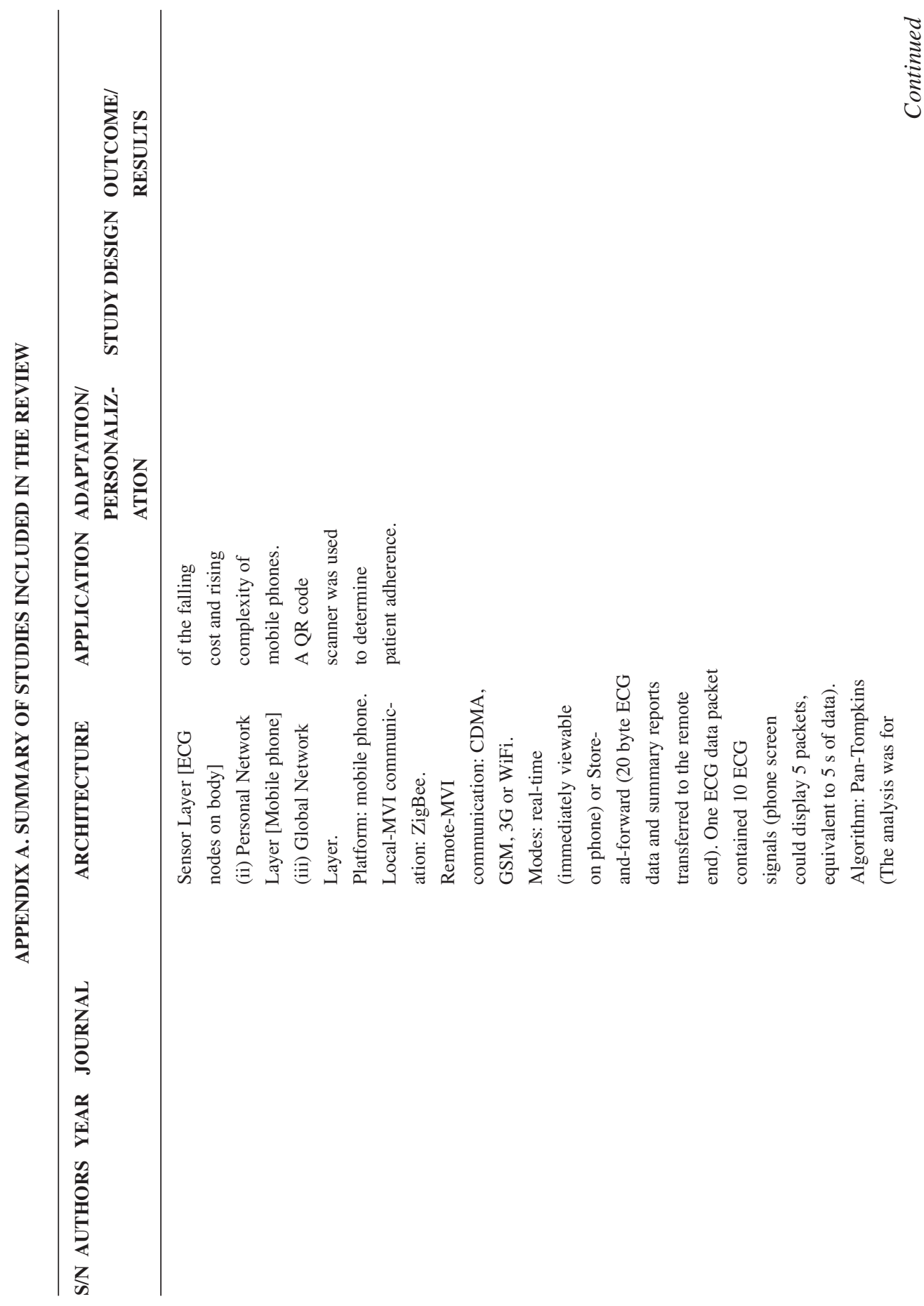




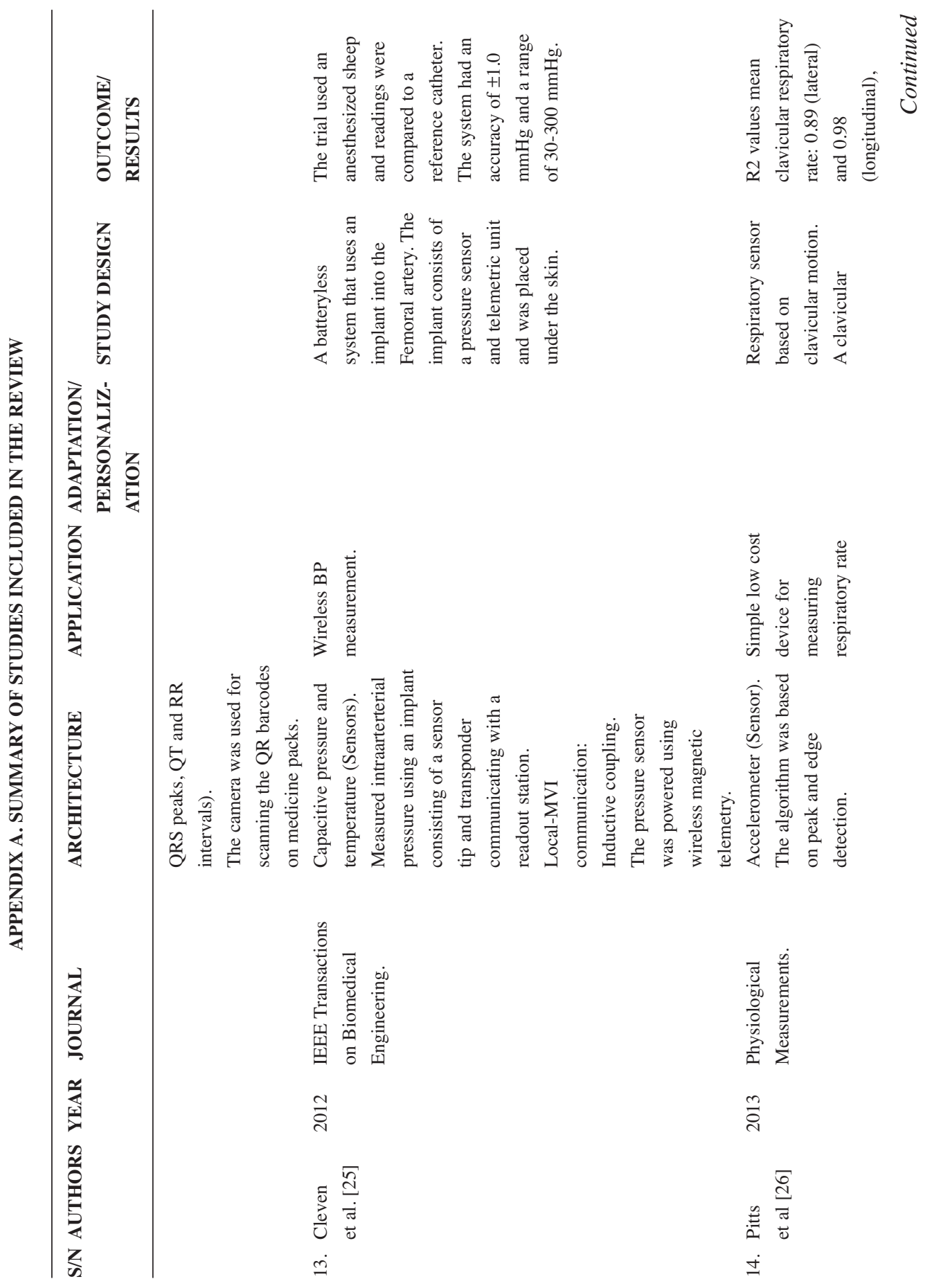




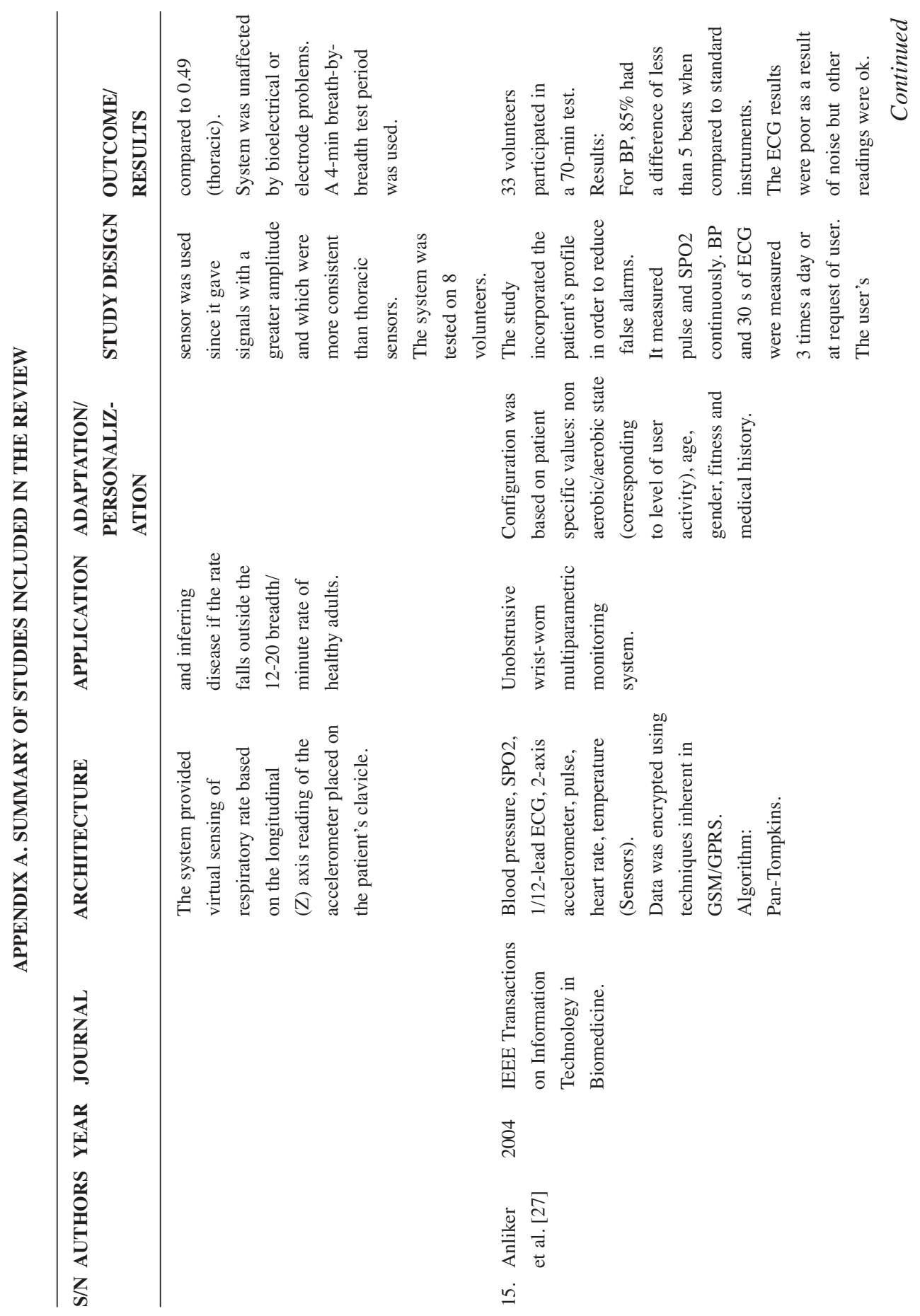




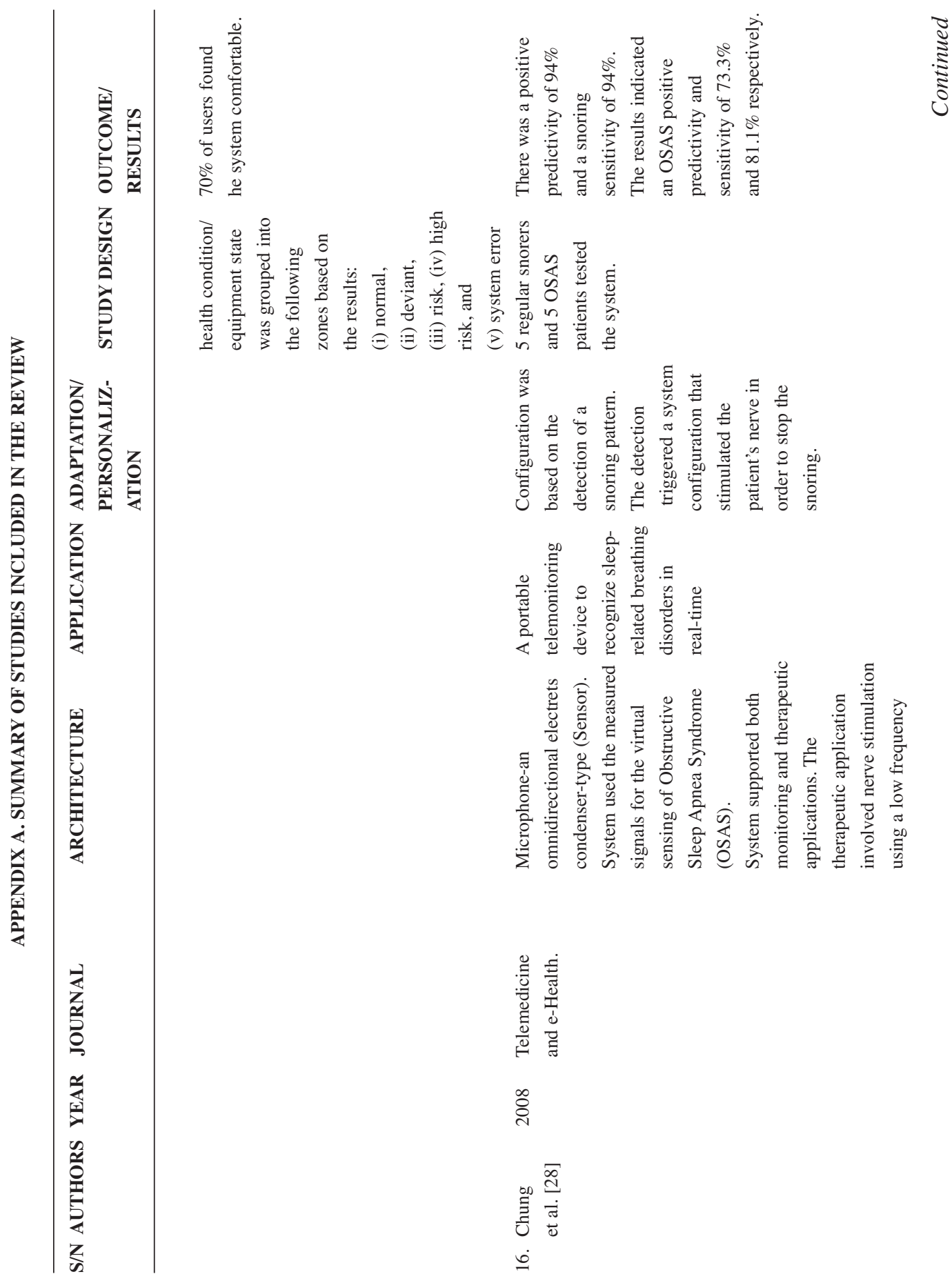




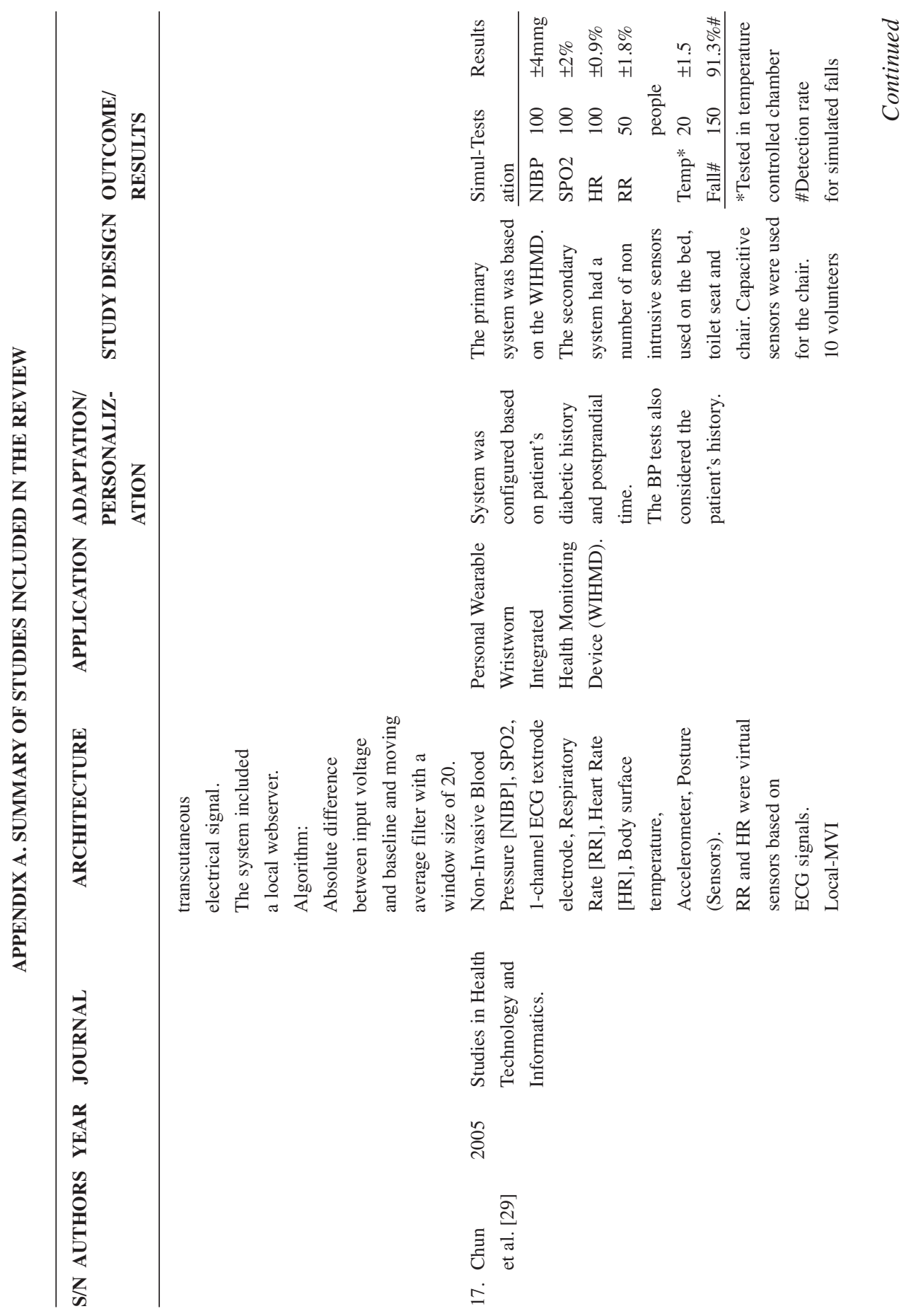




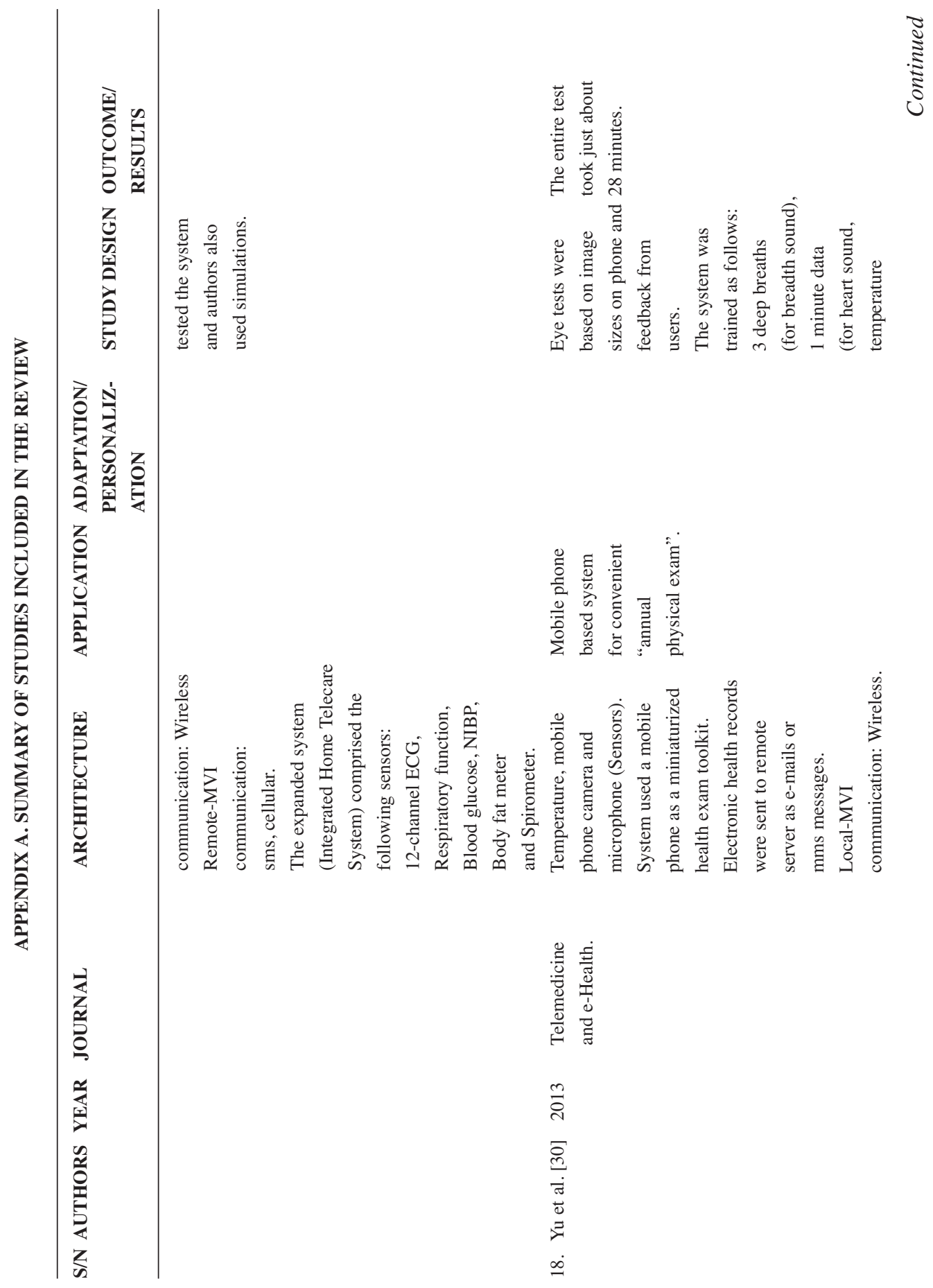




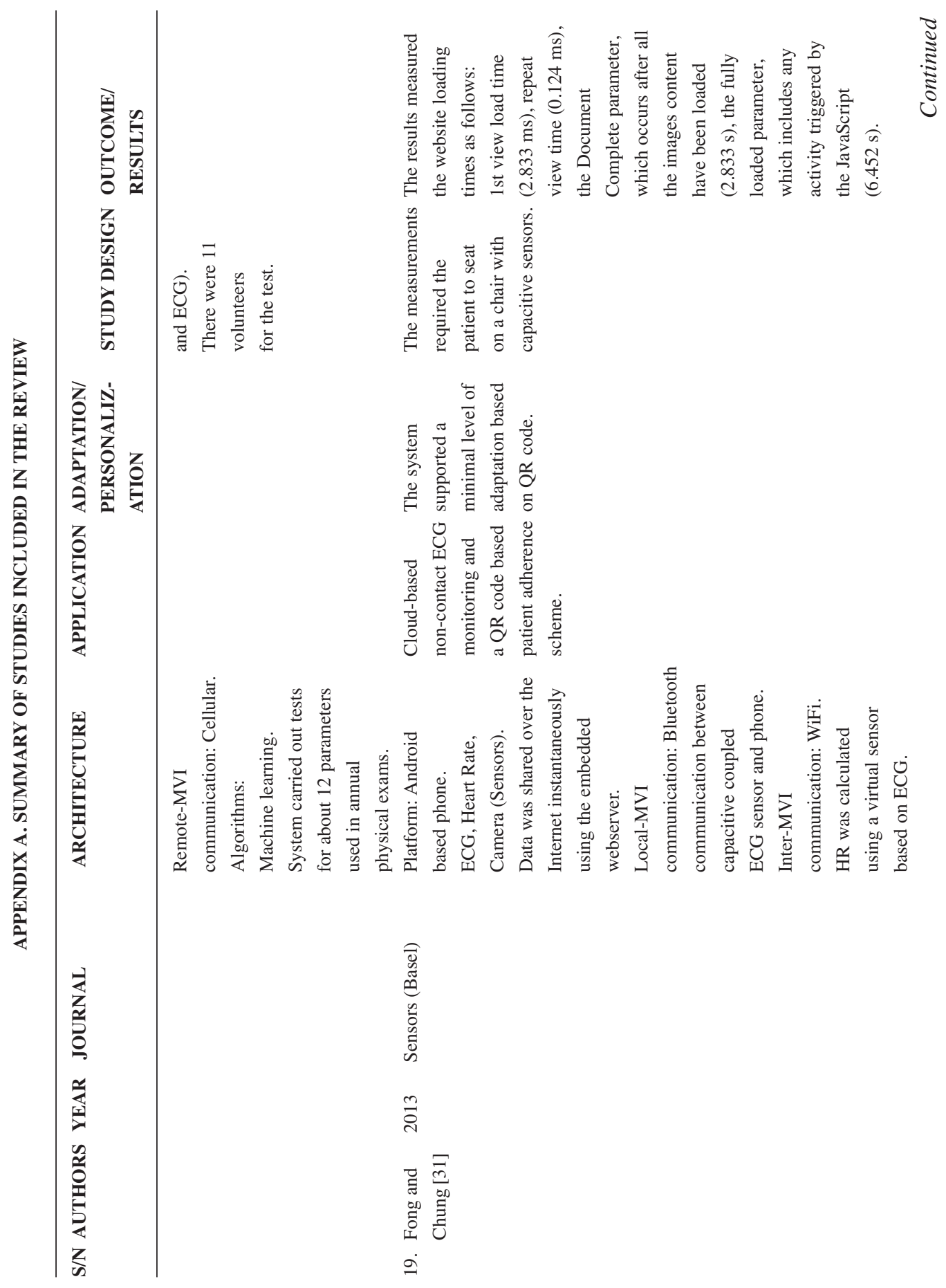




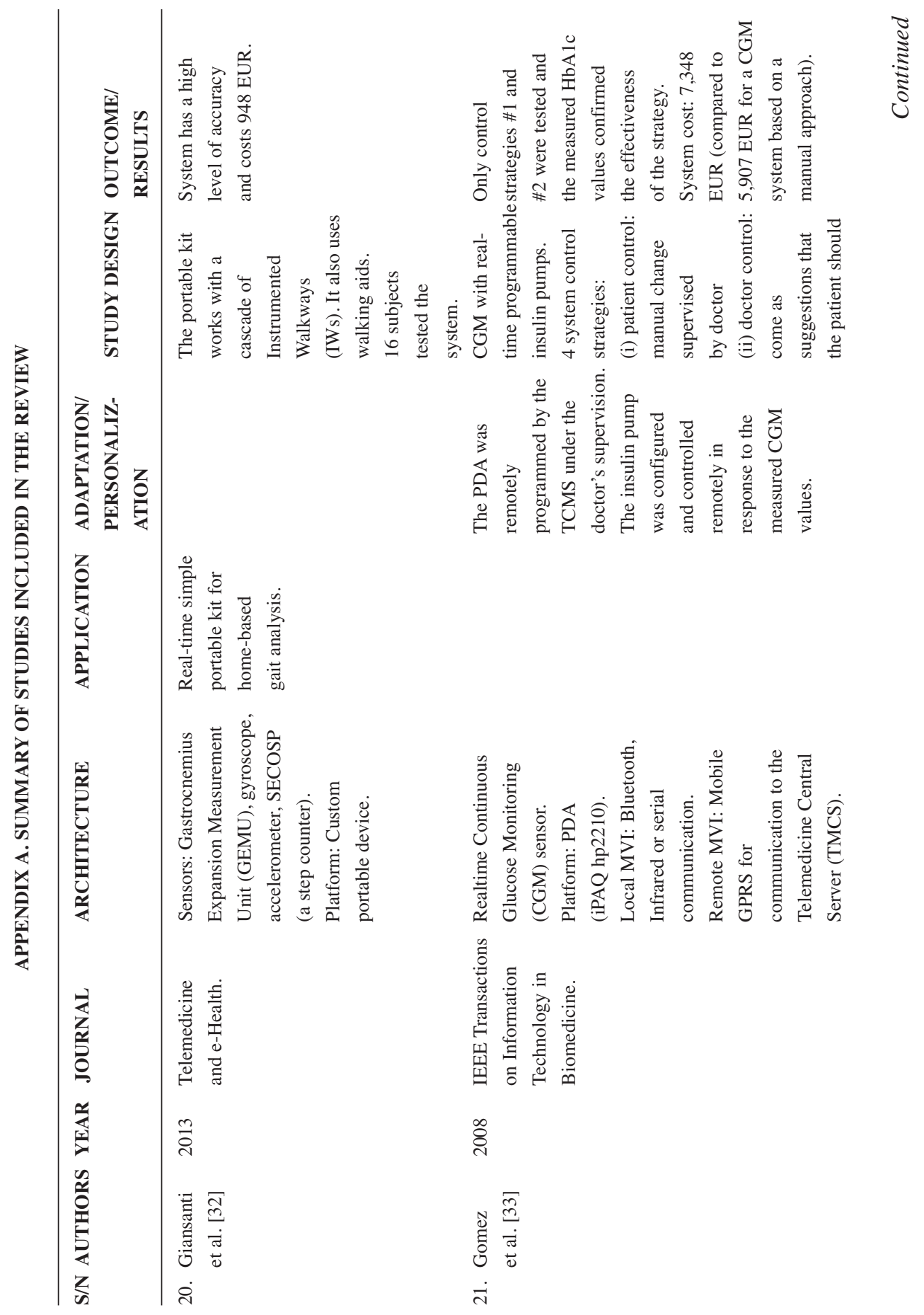




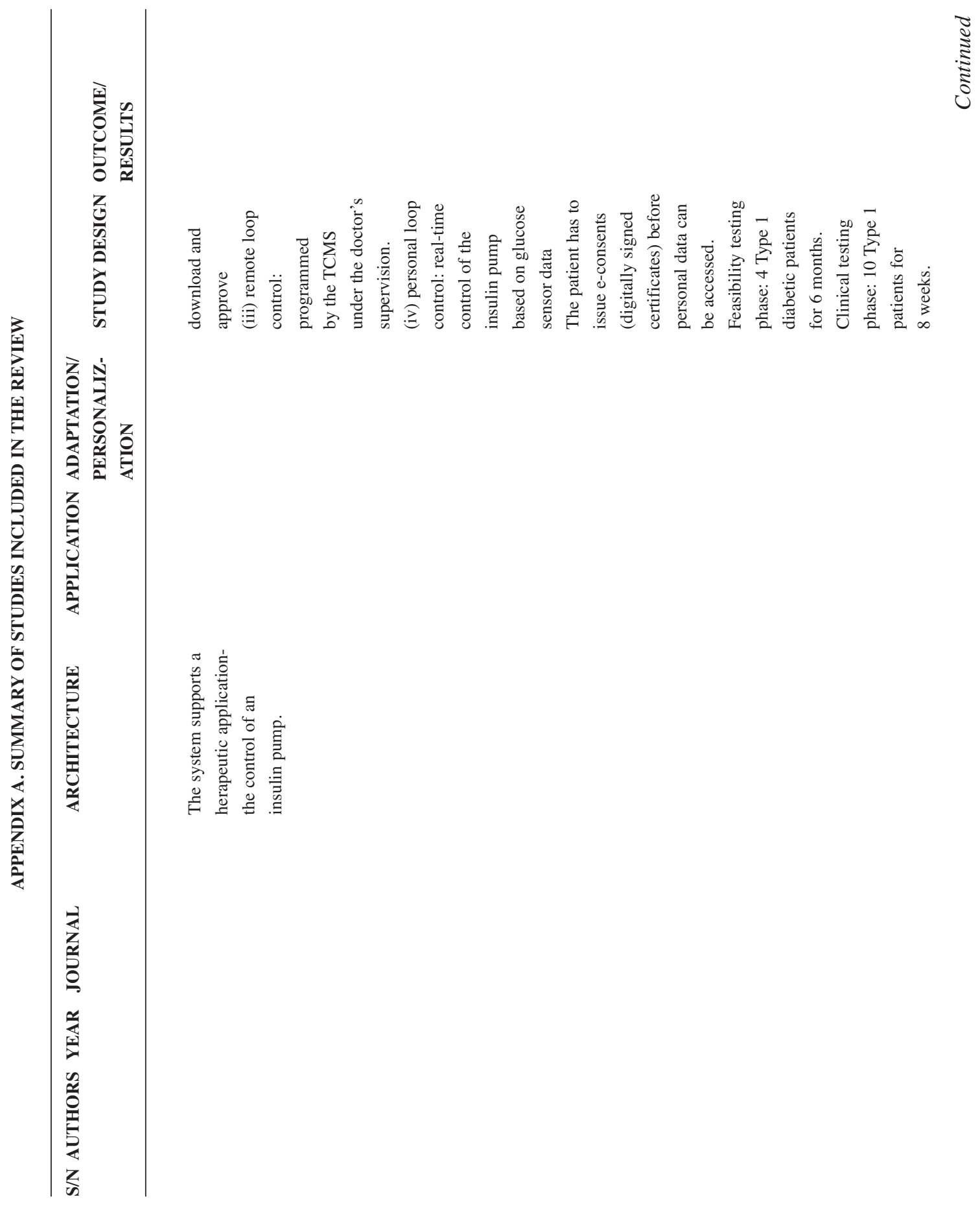




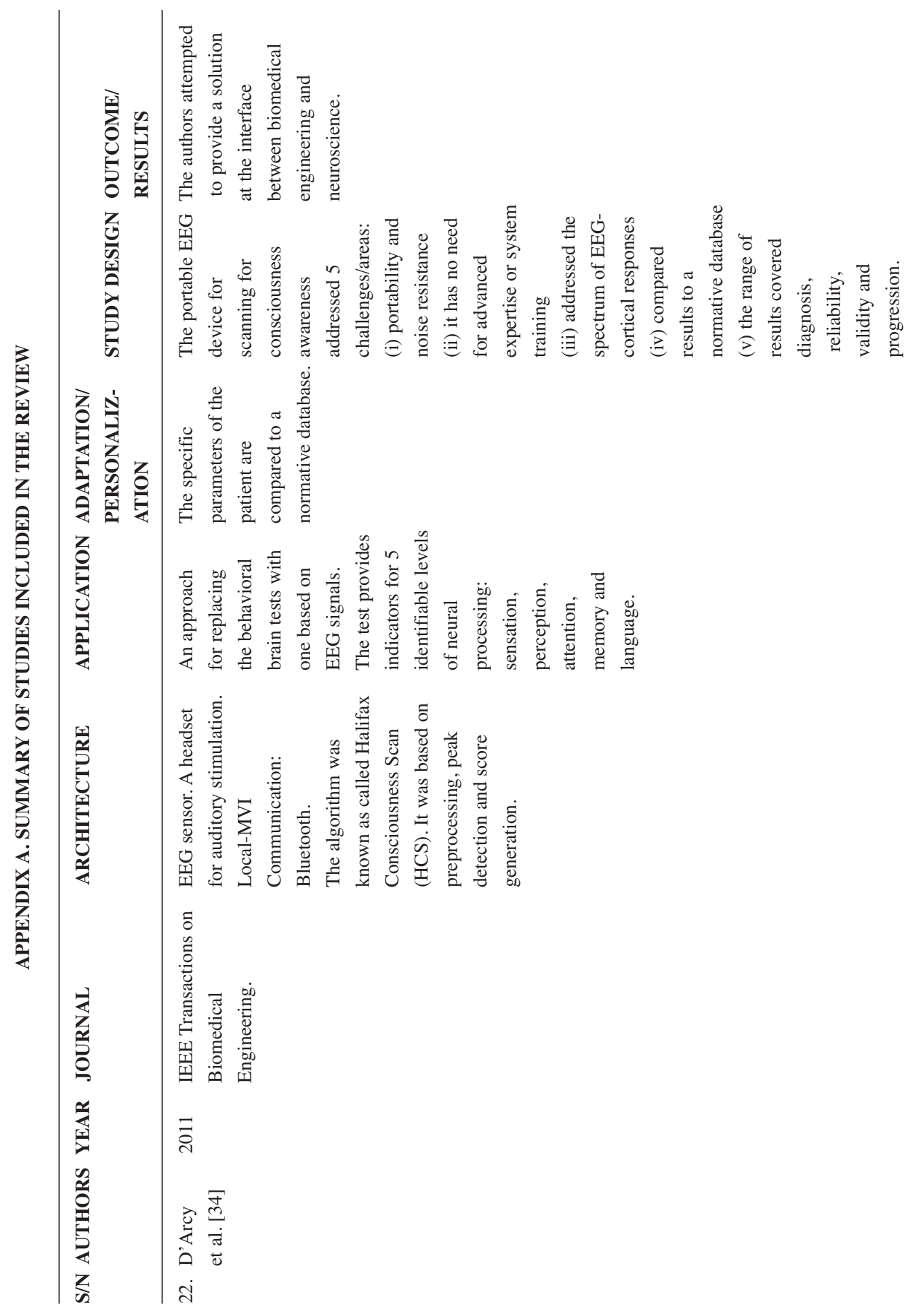




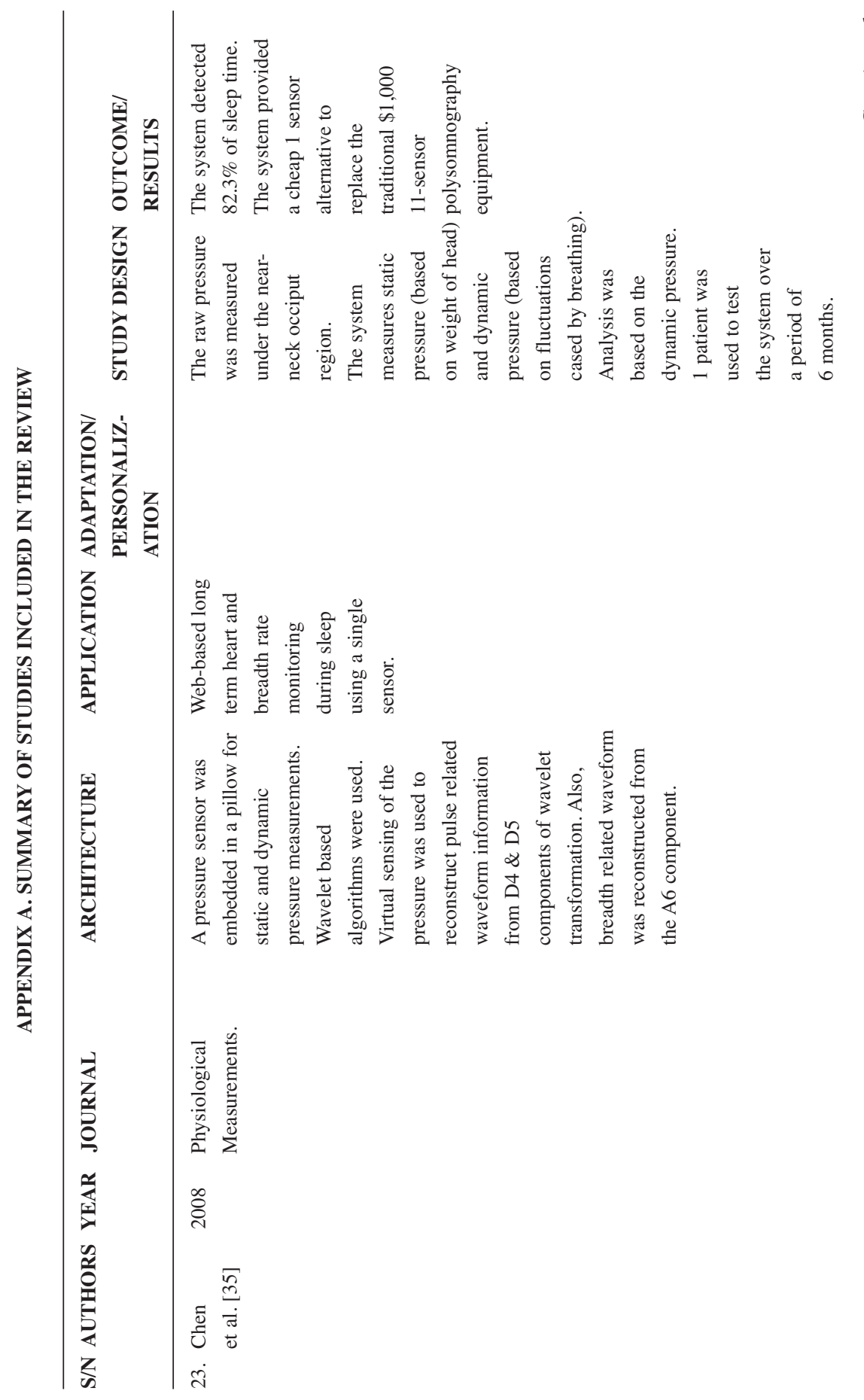




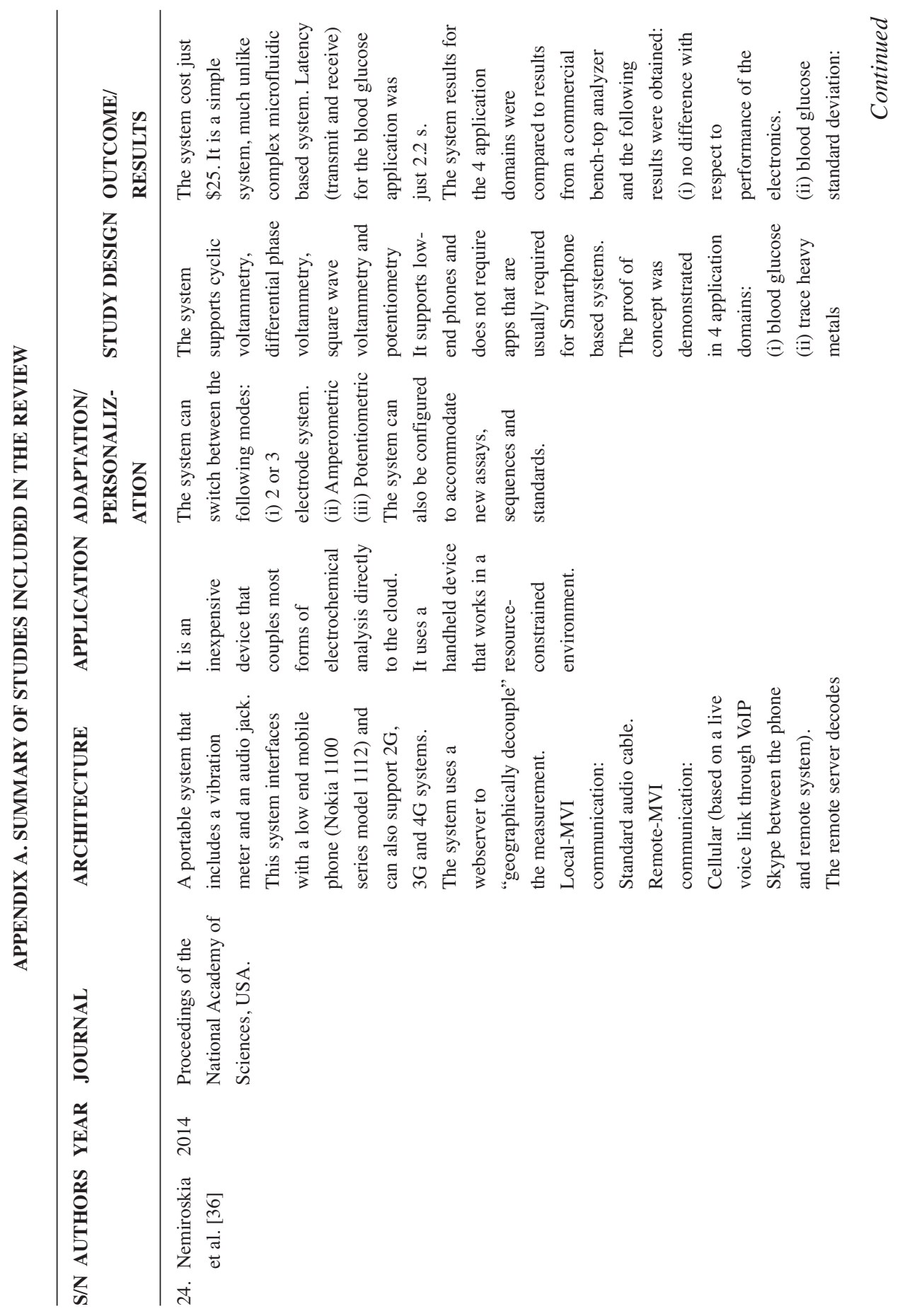




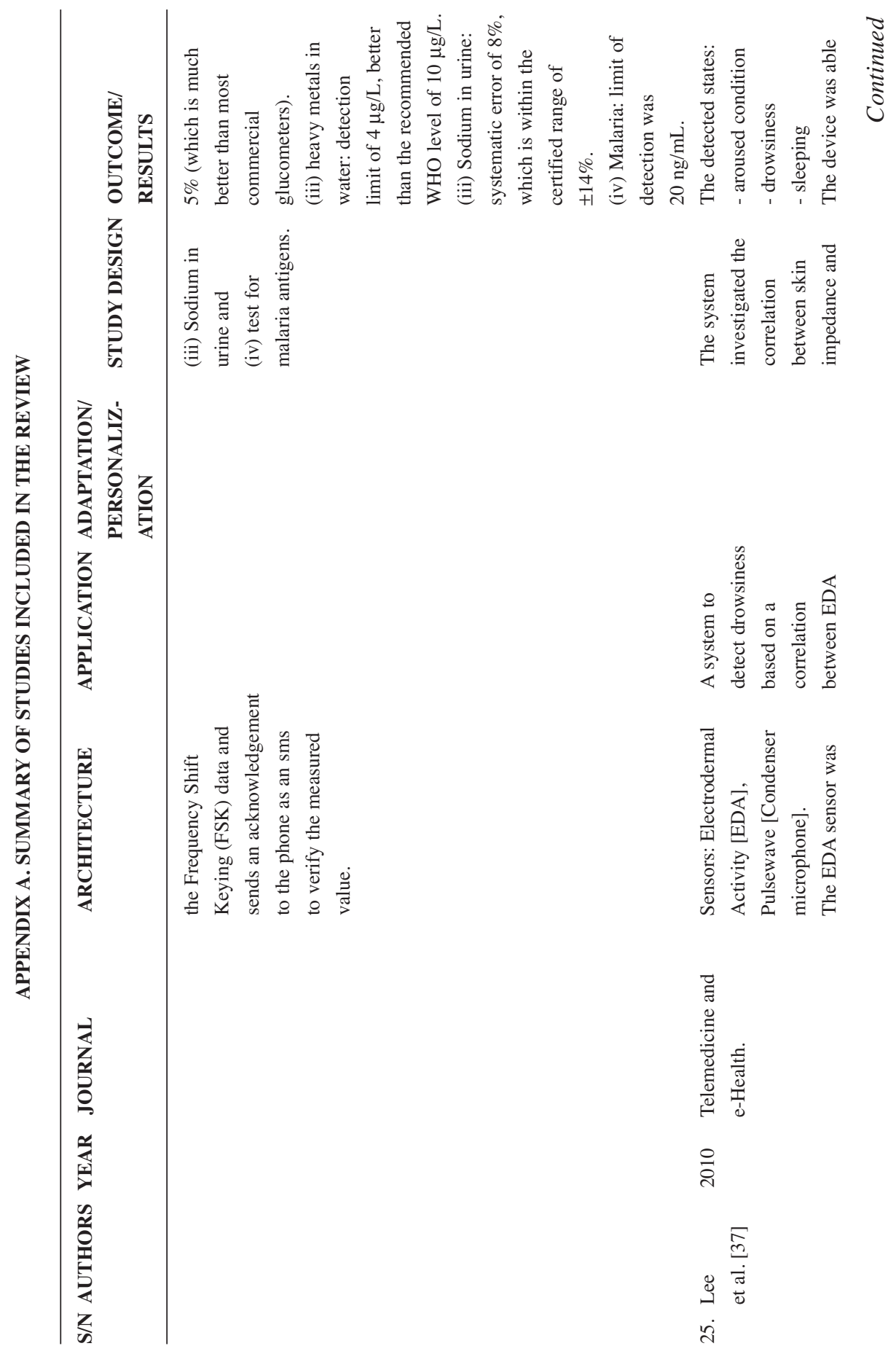




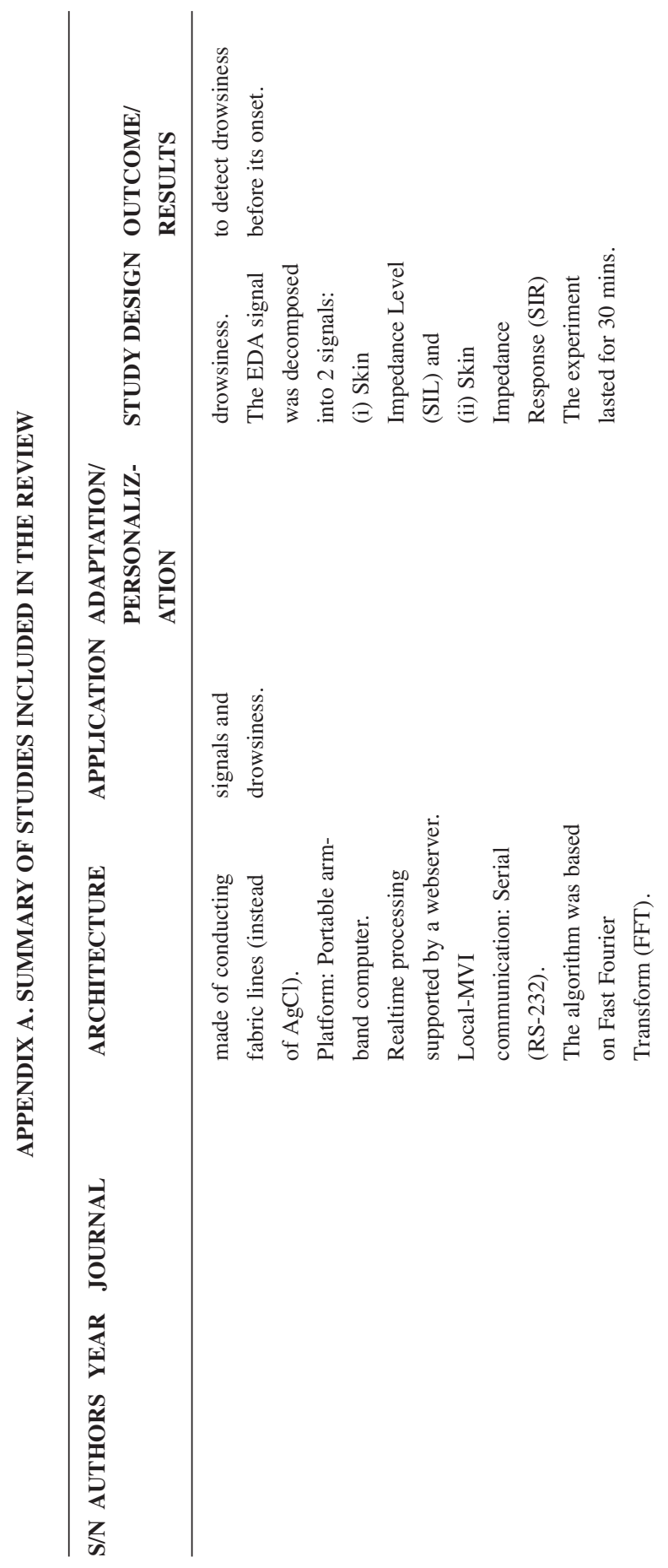





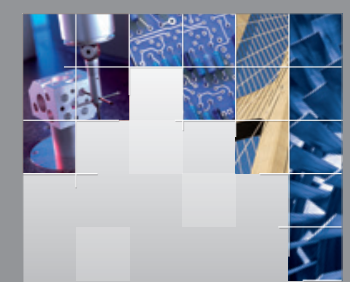

\section{Enfincering}
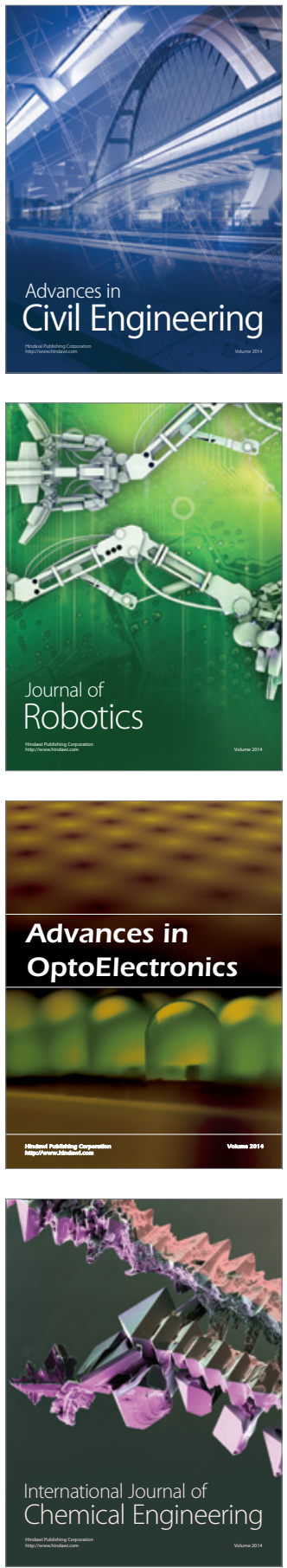

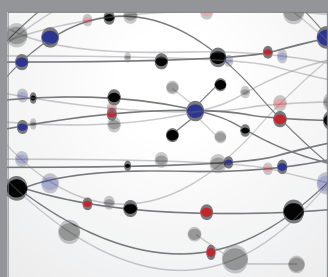

The Scientific World Journal

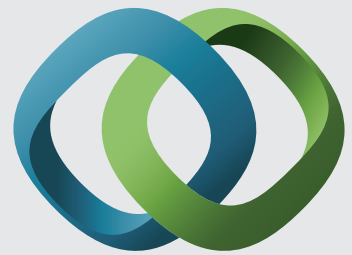

\section{Hindawi}

Submit your manuscripts at

http://www.hindawi.com
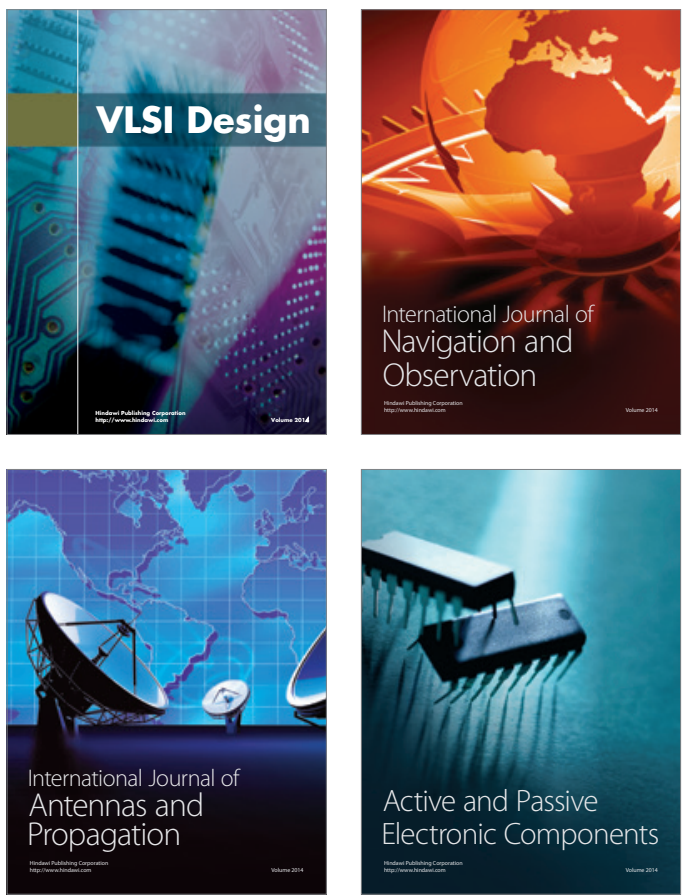
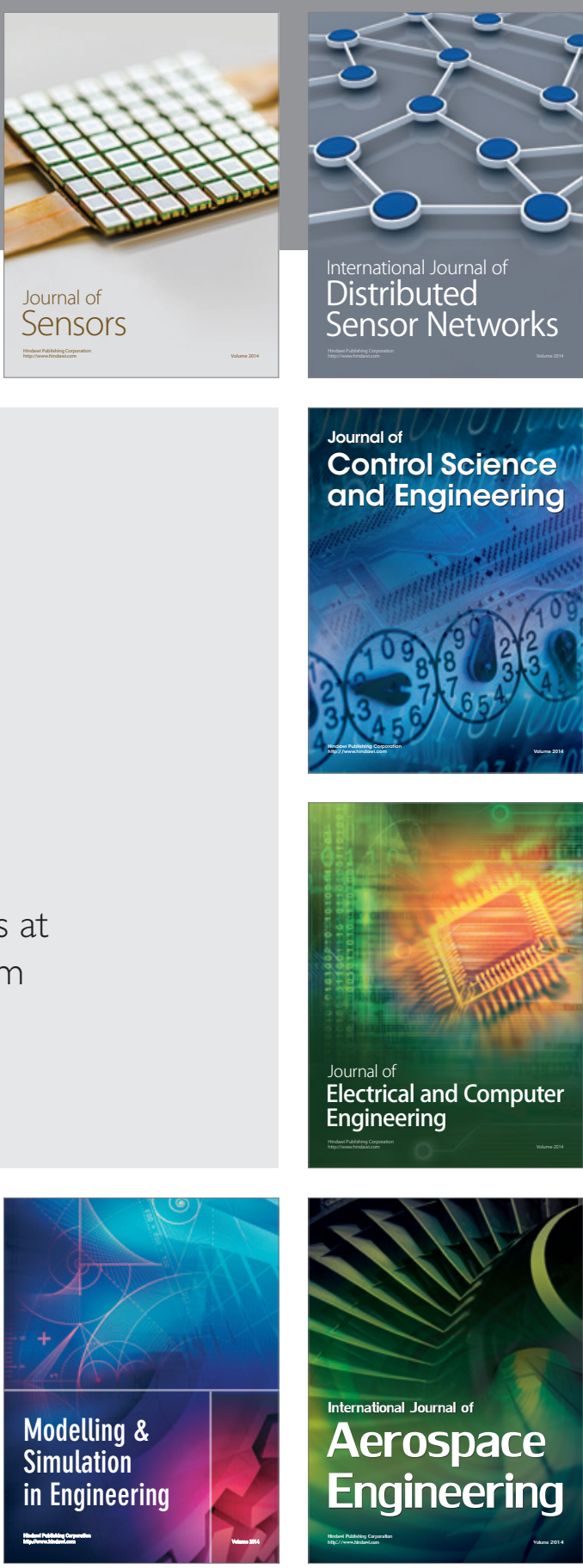

International Journal of

Distributed

Sensor Networks

Journal of

Control Science

and Engineering
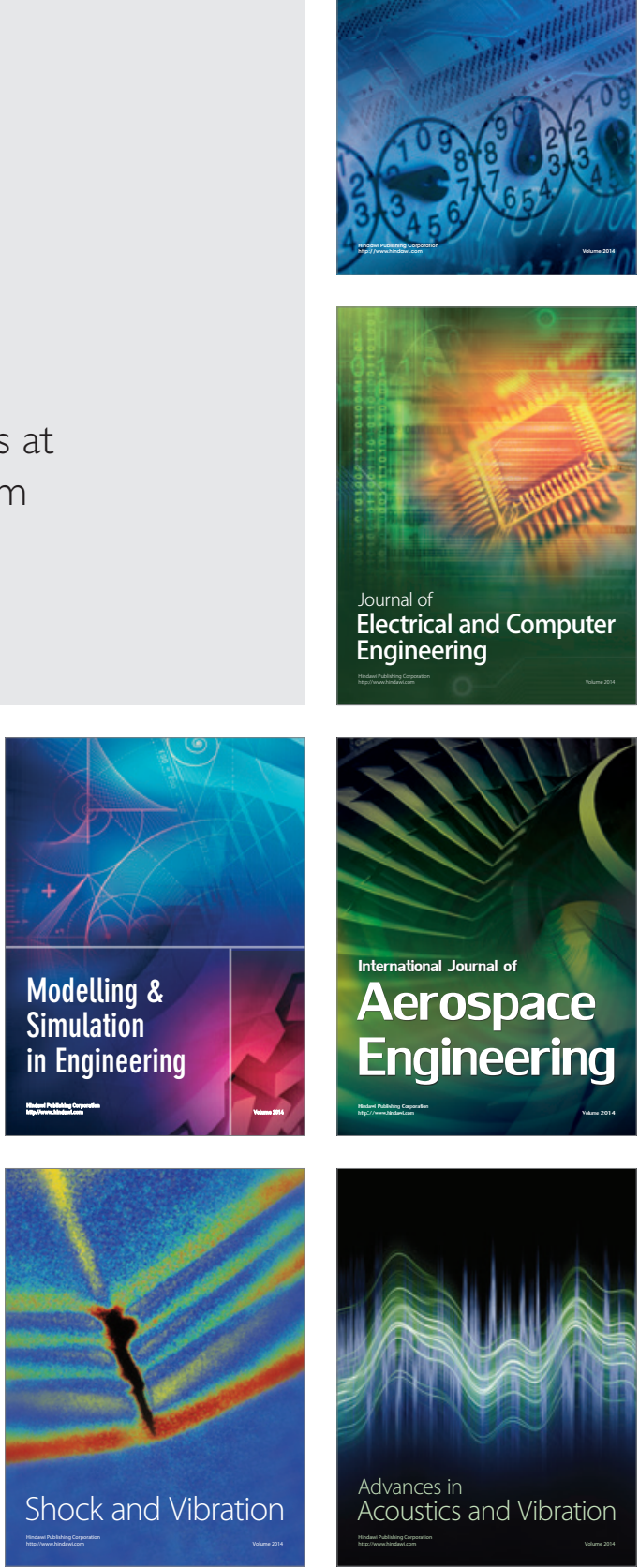